To appear in The Astrophysical Journal, 600, January 1, 2004

Preprint typeset using IATEX style emulateapj v. 11/12/01

\title{
EARLY GROWTH AND EFFICIENT ACCRETION OF MASSIVE BLACK HOLES AT HIGH REDSHIFT
}

\author{
M. VestergaARD \\ Department of Astronomy, The Ohio State University, 140 West 18th Avenue, \\ Columbus, OH 43210-1173. Email: vester@astronomy.ohio-state.edu \\ Accepted by ApJ, Sept. 14, 2003
}

\begin{abstract}
Black-hole masses of the highest redshift quasars $(4 \lesssim z \lesssim 6)$ are estimated using a previously presented scaling relationship, derived from reverberation mapping of nearby quasars, and compared to quasars at lower redshift. It is shown that the central black holes in luminous $z \gtrsim 4$ quasars are very massive $(\gtrsim$ $\left.10^{9} M_{\odot}\right)$. It is argued that the mass estimates of the high- $z$ quasars are not subject to larger uncertainties than those for nearby quasars. Specifically, the large masses are not overestimates and the lack of similarly large black-hole masses in the nearby Universe does not rule out their existence at high- $z$. However, AGN host galaxies do not typically appear fully formed and/or evolved at these early epochs. This supports scenarios in which black holes build up mass very fast in a radiatively inefficient (or obscured) phase relative to the stars in their galaxies. Additionally, upper envelopes of $M_{\mathrm{BH}} \approx 10^{10} M_{\odot}$ and $L_{\mathrm{bol}} \approx 10^{48}$ $\operatorname{ergs~s}^{-1}$ are observed at all redshifts.
\end{abstract}

Subject headings: galaxies: active — galaxies: fundamental parameters — galaxies: high-redshift — quasars: emission lines - ultraviolet: galaxies

\section{INTRODUCTION}

High-redshift quasars are of immediate interest to both cosmologists and active galactic nuclei (AGNs) specialists for several reasons. For example, quasars can be observed out to greater distances and with more ease than quiescent galaxies, owing to their unparalleled energy output over essentially the entire electromagnetic spectrum (e.g., Elvis et al. 1994). Since quasars reside in the central regions of massive galaxies (e.g., Boroson \& Oke 1982; Boroson, Oke, \& Green 1982; Kukula et al. 2001), they provide a valuable beacon to locate galaxies at early times, which is difficult by other means ${ }^{1}$ (Lanzetta et al. 1999). Furthermore, quasar activity is intimately connected with the hierarchical buildup of galaxies (e.g., Haehnelt, Natarajan, \& Rees 1998; Cattaneo, Haehnelt, \& Rees 1999; Ferrarese \& Merritt 2000; Gebhardt et al. 2000a; Haehnelt \& Kauffmann 2000; Colina et al. 2001; Menou, Haiman, \& Narayanan 2001). In fact, quasars possibly play a profound role for the formation and evolution of most, if not all, galaxies (e.g., Kauffmann \& Haehnelt 2000; Milosavljević \& Merritt 2001, 2002; Benson et al. 2002). The quasar phase may even precede the formation of the galaxies themselves (Rix et al. 2001; Kauffmann \& Haehnelt 2000; Omont et al. 2001) and impact their early formation and evolution (e.g., Silk \& Rees 1998; Haiman, Madau, \& Loeb 1999; Theuns, Mo, \& Schaye 2001; Di Matteo et al. 2003).

By virtue of being associated with the highest density peaks in the matter distribution (e.g., Efstathiou \& Rees 1988; Volonteri, Haardt, \& Madau 2002), quasars are an easy probe of these peaks at the earliest epochs and hence of some of the first structures formed in the early Universe (e.g., Haiman \& Loeb 1998). In addition, quasars help probe the nature of the medium in which they are em- bedded. For example, the spectra of $z \approx 6$ quasars place constraints on the epoch of re-ionization (Fan et al. 2002). The presence of luminous, massive ${ }^{2}$ quasars at redshift 6 , when the Universe was less than $10 \%$ of its present age, already place much-needed constraints on models of the earliest structure formation (Turner 1991; Haiman \& Loeb 2001; Volonteri et al. 2002).

High-redshift quasars are also studied (a) to track the growth and evolution of the central black holes (Haiman \& Loeb 2001; Merritt \& Ferrarese 2001a; Ferrarese 2002; A. Steed et al. 2003, in preparation), (b) to understand the central engine (e.g., Peterson, Polidan, \& Pogge 2001; Peterson 2002) responsible for their monstrous energy release, and (c) to understand the evolution of the quasars and active galaxies in general (e.g., Pei 1995; Boyle et al. 2000; see Hartwick \& Schade 1990 and Osmer 2003 for reviews).

It is well established that quasars and their activity reign ${ }^{3}$ between redshift 2 and 3 (Osmer 1982; Hartwick \& Schade 1990; Warren, Hewett, \& Osmer 1994; Schmidt, Schneider, \& Gunn 1995; Kennefick, Djorgovski, \& Meylan 1996; Fan et al. 2001a). The decline in comoving space density above redshifts of $\sim 3.5$ is significant not only for the optically selected quasars but also for the radioselected subset (Shaver et al. 1996; Hook et al. 2002) and for X-ray selected sources (Hasinger 2002); the latter may decline more slowly with increasing redshift, but the statistics are still relatively poor. The question naturally arises whether or not the space density drop toward higher $z$ also signifies a change in cardinal properties of the quasar population at that epoch, given the relatively short time available to generate massive black holes; at $z \gtrsim 3$ the Universe was only $<10 \%$ of its present age for a $\Lambda=0$ Universe (and $\lesssim 15 \%$ of its present age for $\Lambda=0.7$ ).

${ }^{1}$ However, SIRTF, JWST, FIRST/Herschel and ALMA will undoubtedly change this (e.g., Neufeld 1999; Omont 2003).

2 This is typically estimated via the Eddington argument but these high black-hole masses are also confirmed in this work.

${ }^{3}$ Quasars were most numerous at the epoch at $z \approx 2.5$, yet they are still rare objects (e.g., Hartwick \& Schade 1990) relative to the galaxy population (e.g., Huchra \& Burg 1992). 
Recent developments allow virial black-hole masses in distant quasars to be estimated using scaling relationships calibrated to reverberation mapping masses of nearby AGNs and quasars (Wandel, Peterson, \& Malkan 1999; Kaspi et al. 2000; Vestergaard 2002, hereafter Paper I). In this work, these scaling relationships are used to estimate the black-hole masses in high- $z$ quasars. The main focus in this work is to compare the typical central masses of quasars just below and above the epoch $z \approx 3.5$ at which the comoving space density of quasars declines. In addition, these masses are discussed in light of our current knowledge of the nature of their host galaxies. Representative quasar samples in the redshift range $1.5<z<6.3$ are studied $(\S 3)$, including part of the large, high-quality data bases of high-redshift quasars now available. Several surveys have in the past $\sim 10$ years turned up a large number of previously unknown $z \approx 4$ quasars (e.g., Kennefick et al. 1995a, 1995b, 1996; StorrieLombardi et al. 1996; Schneider, Schmidt, \& Gunn 1997; see also Constantin et al. 2002). The Sloan Digital Sky Survey (SDSS) has in recent years had particular success in uncovering an even larger number of quasars at redshifts greater than 3.5 (Fan et al. 1999, 2000, 2001b, 2001c; Anderson et al. 2001). For a large subset of these quasars for which spectra are published, the black-hole masses were estimated in this work and compared with mass estimates of lower-redshift quasars, namely the well-selected $z \approx 2$ quasar sample of Vestergaard (2000). Given current and earlier survey capabilities, these samples necessarily consist of luminous quasars. Since data on nearby luminous quasars and AGNs readily exist in the Bright Quasar Survey sample (hereafter BQS; Schmidt \& Green 1983), this sample was also included for comparison and reference. The lack of objects between $0.5 \leq z \leq 1.5$ does not affect the conclusions of this study.

The structure of this paper is as follows. The approach in estimating $M_{\mathrm{BH}}, L_{\mathrm{bol}}$, and $L_{\mathrm{bol}} / L_{\mathrm{Edd}}$ is outlined next $(\S 2)$. The data are described in $\S 3$, presented in detail in $\S 4$, and discussed in $\S 5$. In particular, since the central masses are very large $\left(\sim 10^{9} M_{\odot}\right)$ for the $z \gtrsim 4$ quasars, the efficacy of the scaling relations is examined in $\S 5.1$; it is concluded that the scaling relations are fully applicable to the high- $z$ quasars with an uncertainty of a factor $\lesssim 4$. Section 5.2 discusses the fact that although the masses of these high- $z$ active black holes are rather large, the AGN host galaxies at $z \gtrsim 4$ appear to be rather young, and even small, young galaxies at $z \approx 3$ are capable of hosting massive black holes. This leads to the conclusion that super-massive black holes were in place long before the bulk of the stellar mass in the quasar host galaxies. A summary and conclusions are provided in $\S 6$.

A cosmology with $H_{0}=75 \mathrm{~km} \mathrm{~s}^{-1} \mathrm{Mpc}^{-1}$, $\mathrm{q}_{0}=0.5$, and $\Lambda=0$ is used throughout, although the main conclusions of this study do not depend on the specific choice of cosmology. However, it is worth mentioning that adoption of a cosmology of $H_{0}=70 \mathrm{~km} \mathrm{~s}^{-1} \mathrm{Mpc}^{-1}, \Omega_{M}=0.3, \Omega_{\Lambda}=$ 0.7 will increase the mass estimates at the highest redshifts by no more than a factor 2.4 and by a decreasing factor at lower redshifts.

\section{2. $\mathrm{M}_{\mathrm{BH}}, \mathrm{L}_{\mathrm{bol}}$, AND $\mathrm{L}_{\mathrm{bol}} / \mathrm{L}_{\mathrm{Edd}}$ MEASUREMENTS}

The samples of quasars studied here cover the redshift ranges $z \leq 0.5$ and $1.5 \lesssim z \lesssim 6.3$. Therefore, the observed optical spectra cover restframe wavelengths including $\mathrm{H} \beta$ for the BQS and the region around CIV for the higher redshift quasars.

The virial masses, $M \propto v^{2} R$, are estimated from singleepoch spectral measurements using the relationships presented in Paper I (eqns. 1, 2, A5, and 7), since they are relevant for spectroscopic data covering the wavelength regions around the $\mathrm{H} \beta$ and $\mathrm{C}$ IV lines. Here $v$ is the Doppler width of an emission line and $R$ is the radius of the lineemitting region. Note that also the high-ionization lineemitting gas, including $\mathrm{C}$ IV, yields virial masses consistent with those derived from $\mathrm{H} \beta$ in all the objects for which this can be tested (Peterson \& Wandel 2000; Onken \& Peterson 2002). Hence, the C IV line width is valid as a measure of $v$ (see discussion in $\S 5.1$ ).

The important scaling relationship for the mass equations listed below is the radius - luminosity relation, that allows the size of the broad line region (BLR) to be estimated from the optical continuum luminosity of the source. The empirical scaling law, applicable to Balmer lines and based on reverberation-mapped nearby AGNs and quasars, is (Kaspi et al. 2000):

$$
R_{\mathrm{BLR}}=(32.9 \pm 5.5)\left[\frac{\lambda L_{\lambda}(5100 \AA)}{10^{44} \operatorname{ergs~s}^{-1}}\right]^{0.7 \pm 0.1} \quad \mathrm{lt}-\text { days. }
$$

The mass equation based on optical data (hereafter, the 'optical relationship') is

$$
\begin{aligned}
& \log M_{\mathrm{BH}} / M_{\odot}=6.7+ \\
& \log \left[\left(\frac{\mathrm{FWHM}(\mathrm{H} \beta)}{1000 \mathrm{~km} \mathrm{~s}^{-1}}\right)^{2}\left(\frac{\lambda L_{\lambda}(5100 \AA)}{10^{44} \mathrm{ergs} \mathrm{s}^{-1}}\right)^{0.7}\right]
\end{aligned}
$$

and the mass equation relevant for UV data (the ' $U V$ relationship') is

$$
\begin{aligned}
& \log M_{\mathrm{BH}} / M_{\odot}=6.2+ \\
& \log \left[\left(\frac{\mathrm{FWHM}(\mathrm{C} \mathrm{IV})}{1000 \mathrm{~km} \mathrm{~s}^{-1}}\right)^{2}\left(\frac{\lambda L_{\lambda}(1350 \AA)}{10^{44} \mathrm{ergs} \mathrm{s}^{-1}}\right)^{0.7}\right],
\end{aligned}
$$

where the sample standard deviation of the weighted mean is 0.45 and shows the intrinsic scatter in the sample ( $\mathrm{Pa}$ per I). This is confirmed by the black-hole masses of reverberation mapped AGNs which exhibit an intrinsic scatter of about $0.3-0.5$ dex (Peterson 2002) around the $M_{\mathrm{BH}}-\sigma$ relationship, established by local quiescent galaxies (e.g., Ferrarese \& Merritt 2000; Gebhardt et al. 2000a; see also Gebhardt et al. 2000b and Ferrarese et al. 2001 for comparisons of some AGN masses with the $M_{\mathrm{BH}}-\sigma$ relation). Both the optical and the UV relationships were calibrated (Paper I) to virial mass estimates of low-redshift active galaxies determined from reverberation-mapping analysis.

The velocity dispersion, $v$, of the broad line gas is estimated using the broad emission line width as characterized by FWHM. While equation (1) is valid for the 
Balmer lines only, a similar scaling law, $R_{\mathrm{BLR}} \propto L^{0.7}$, is assumed for the UV relationship; the constant of proportionality is determined by the calibration and included in equation (4). Note that when applying these scaling relationships to higher- $z$ quasars, a large extrapolation in luminosity is not performed, which might otherwise raise concern. The efficacy of the mass scaling relationships is discussed in $\S 5.1$.

The bolometric luminosities are estimated based on existing average spectral energy distributions (SEDs). SEDs are only available for a small group of distant quasars. Kuhn et al. (2001) found no significant differences between the restframe optical and UV energy distributions for 15 $z>3$ quasars and low- $z$ quasar SEDs. It is fair to assume the Kuhn et al. sample is representative, also of quasars at higher redshifts yet, given the similarity of quasar spectra at all redshifts (see $\S 5$ ). Specifically, this means that the empirical average SED of low- $z$ active galaxies and quasars (Elvis et al. 1994) is also representative of the average SED of more distant quasars. Here, the average radio-quiet quasar (RQQ) SED of Elvis et al. (1994) is updated to reflect the more representative optical-to-X-ray slope of 1.43 determined by Elvis, Risaliti, \& Zamorani (2002) using new and improved X-ray data. This updated RQQ SED was used to estimate bolometric correction factors applicable also to radio-loud quasars (RLQs), because (1) high- $z$ RLQs appear to constitute a similar low fraction $(\sim 10 \%)$ of all quasars as seen for $z \lesssim 2$ quasars (Stern et al. 2000), and (2) the average RLQ SED has not yet been updated (M. Elvis, 2002, private communication). The bolometric correction factors to the monochromatic continuum luminosities, $\lambda L_{\lambda}$, at rest wavelengths of $1350 \AA, 1450 \AA$, and $4400 \AA$ were computed ${ }^{4}$ to be $4.62,4.65$, and 9.74 , respectively. Applying a bolometric correction to the UV continuum luminosities for the high- $z$ quasars reduces the uncertainty in the $L_{\text {bol }}$ estimate compared to using a bolometric correction to luminosities at other wavelengths. The reasons are that one eliminates the uncertainty associated with the measured UV continuum slope and extrapolation to other wavelengths, and since $\lambda 1350$ and $\lambda 1450$ are close to the peak of the big blue bump, the associated bolometric correction factors are the smallest relative to other wavelengths.

The conservative measurement uncertainties estimated from the line widths, continuum fluxes, and continuum slopes were propagated to estimate the measurement errors on $M_{\mathrm{BH}}, L_{\mathrm{bol}}$, and the Eddington ratios $L_{\mathrm{bol}} / L_{\mathrm{Edd}} \propto$ $L_{\mathrm{bol}} / M_{\mathrm{BH}}$. These uncertainties do not include the uncertainty associated with the use of bolometric corrections or the use of the simple scaling relationships in eqn. (3), (1), and (4) to estimate the $M_{\mathrm{BH}}$. A statistical $1 \sigma$ uncertainty of about 0.4 dex and 0.5 dex (i.e., a factor 2.5 and 3 in the mass) for the optical and UV relationships, respectively, was found for the sample studied in Paper I. This additional uncertainty must be kept in mind, especially when considering the masses in absolute terms. However, since
$M_{\mathrm{BH}}$ can likewise be reasonably estimated for high redshift quasars ( $§ 5.1)$, statistical analysis and comparison of the relative masses of high-redshift quasars should be reasonably sound.

\section{DATA}

Several samples of quasars at low, intermediate, and high redshift are analyzed here as described in detail below.

\subsection{Low Redshift Quasars}

This sample consists of the 87 BQS quasars at $z \leq$ 0.5 . For the objects with available reverberation-mapping masses, these masses are used, as computed in Paper I. For the remaining sources, the masses are estimated from equation (3). The measurements of line widths and continuum luminosities used are explained in detail in Paper I. Briefly, the $\mathrm{FWHM}(\mathrm{H} \beta)$ measurements were primarily adopted from Boroson \& Green (1992). The monochromatic luminosities, $L_{\lambda}(5100 \AA)$, were determined for most of the objects from the spectrophotometry by Neugebauer et al. (1987), but for a few sources $L_{\lambda}(5100 \AA)$ was computed from $4400 \AA$ flux densities (Kellerman et al. 1989) by adopting a power-law slope, $\alpha=-0.5\left(\mathrm{~F}_{\nu} \propto \nu^{\alpha}\right)$. Bolometric luminosities for different subsets of the BQS quasars have been measured by a few authors (Sanders et al. 1989; Elvis et al. 1994; Lonsdale, Smith, \& Lonsdale 1995; Wilkes et al. 1999a). Comparison of the measurements for quasars common between two or more of these studies shows that the $L_{\text {bol }}$ values are generally relatively similar ${ }^{5}$ within a common scatter of $0.2-0.3 \mathrm{dex}$, consistent with the estimated measurement uncertainties quoted by Elvis et al. (1994). In the interest of simplicity and consistency with the data used for the quasar samples at higher-redshifts, the $L_{\mathrm{bol}}$ values were here estimated as $(9.74 \pm 4.3) \times \lambda L_{\lambda}(4400 \AA)(\S 2)$. Adopting this scaling introduces an uncertainty similar to the measurement uncertainties quoted above and is thus considered acceptable. Errors on the parameters are propagated using the measurement uncertainties on the primary measurements as described in Paper I.

\subsection{Intermediate Redshift Quasars}

The sample of 114 quasars at intermediate redshifts $(1.5 \lesssim z \lesssim 3.5)$ studied here is that analyzed by Vestergaard (2000). The sample consists of 68 RLQs and 48 RQQs. The RLQs were selected from Barthel et al. (1988), Barthel, Tytler, \& Thomson (1990), Murphy, Browne, \& Perley (1993), and the 3C and 4C radio catalogs to span the range of observed quasar radio spectral indices; this allows a study of how the spectral features may change with source inclination with respect to our line of sight (e.g., Vestergaard, Wilkes, \& Barthel 2000). Most of the RLQ data are from Barthel et al. (1990). The individual RQQs were selected from Hewitt \& Burbidge

\footnotetext{
4 The bolometric correction to $\lambda L_{\lambda}(4400 \AA)$ of 9.74 is slightly lower than the average correction of 11.8 established by Elvis et al. (1994). The reason is in part the weaker contribution from the X-rays, and in part due to the use of the average SED in this work compared to the average of the individual bolometric correction factors quoted by Elvis et al. (1994). The scaling factor of 9.74 is preferred for $\lambda L_{\lambda}(4400 \AA)$ for consistency with the $1350 \AA$ and $1450 \AA$ bolometric correction factors which were not quoted by Elvis et al.

5 While one can argue that one of the studies has better X-ray data, then another has better IR data, and for individual objects, the SED of one study may be preferred over that of another. However, it is beyond the scope of this work to compile updated SEDs based on the best data now available.
} 
Vestergaard

(1993) to be well-matched and paired in redshift and luminosity to individual quasars in the radio-loud subset. The purpose was to study the rest UV spectral characteristics with radio properties (Vestergaard 2000). Given the careful sample selection, this sample of 116 quasars is here considered reasonably representative of the $z \approx 2$ quasar population to be compared with the higher redshift quasars. The full sample, as opposed to the pair-matched subset, is studied here to improve the statistical significance of the comparison.

The CIV line width, FWHM(CIV), was measured reliably for this sample on a smooth representation of the emission profile, obtained by modeling with 2 to 3 Gaussian functions (following Laor et al. 1994), above a global power-law continuum fit to line-free windows (e.g., Francis et al. 1991; Vestergaard \& Wilkes 2001). The FWHM uncertainties were estimated by varying the continuum fit within the continuum flux rms and remeasuring the FWHM. The continuum fluxes were measured from the best fit power-law and the flux uncertainties were determined as the rms scatter around this fit in the continuum fitting windows. The bolometric luminosity is estimated from $L_{\lambda}(1350 \AA)$, i.e., $L_{\mathrm{bol}}=4.62 \times \lambda L_{\lambda}(1350 \AA)[\S 2]$.

\subsection{High Redshift Quasars}

The high- $z$ quasar sample consists of $\sim 150 z>3.5$ quasars, mostly from the SDSS, selected from recently published work, as detailed below. For consistency, the spectra of these quasars were measured in a similar manner, as follows: FWHM(C IV ) was measured relative to a reasonable, best-fit continuum determined using virtually line-free continuum windows. The continuum flux at rest frame wavelengths $1350 \AA$ and $1450 \AA$ were measured from this best-fit power-law continuum. Conservative measurement uncertainties on line widths, continuum fluxes, and spectral slopes were estimated based on the continuum $\mathrm{rms}$ noise, as described above for the intermediate- $z$ quasars. The spectral slope measured here is only used when more accurate, published values are unavailable. $L_{\text {bol }}$ was estimated as $4.65 \times \lambda L_{\lambda}(1450 \AA)(\operatorname{see} \S 2)$.

The source studies from which the individual quasars are drawn are listed below. In each case, the details of the analysis specific to the individual data set are outlined.

Constantin et al. (2002): High-quality spectra of these $z \approx 4$ quasars were kindly provided by the authors. Constantin et al. corrected their data for Galactic reddening using Schlegel, Finkbeiner, \& Davis (1998) $A_{V}$ values and the Cardelli, Clayton, \& Mathis (1989) extinction curve. Constantin et al. quote an uncertainty of 0.2 dex in their $\mathrm{AB}(1450 \AA)$-based, monochromatic luminosities. The $L_{\lambda}(1450 \AA)$ values measured here were recalibrated to match the AB-based $L_{\lambda}(1450 \AA)$ for the few objects showing a difference larger than 0.2 dex between the two sets of luminosity measurements. The spectra of 40 of the 44 quasars could be measured. A few quasars are too strongly absorbed in the C IV emission profile for a reasonable measurement of the line width. One quasar exhibited no conspicuous emission lines other than Ly $\alpha$ and $\mathrm{N}$.

Anderson et al. (2001): The digital SDSS spectra of these $\sim 125$ quasars are publicly available. The best qual- ity spectra were selected for measurement. A few other poorer quality spectra were also measured. The spectra were first corrected for Galactic reddening using the Schlegel et al. (1998) $E(B-V)$ values obtained from NED and the Cardelli et al. (1989) reddening curve. For a subset $(\sim 60)$ of the quasars Fan et al. (1999, 2000, 2001b) present $\mathrm{AB}(1450 \AA)$ magnitudes to which the spectral measurements were recalibrated.

Fan et al. (1999, 2000, 2001b): The best-quality spectra (kindly provided by X. Fan) of these SDSS quasars that include a full, measurable C IV line profile were selected for measurement. The continuum luminosities measured here at $1350 \AA$ and $1450 \AA$ were recalibrated to the (dereddened) $\mathrm{AB}(1450 \AA)$ magnitudes. Fan et al. define 3 quasar subsamples. The set of 59 quasars analyzed here (hereafter the "Fan et al. sample") is drawn from these subsamples, as follows: (a) 34 of the 39 quasars that comprise the well-defined "color-selected sample" $(3.6 \lesssim z \lesssim 5$; $i^{*} \leq 20 \mathrm{mag}$ ), complete within the survey area (see Fan et al. 2001b for details), (b) 12 quasars from the "bright subsample" $\left(i^{*} \lesssim 20.2 \mathrm{mag}\right)$ that are not included in (a), and (c) 13 of the 18 "faint subsample" quasars $\left(i^{*}<21\right.$ mag). As mentioned, the selection is mostly based on data quality (but see below). Ten quasars were common between the originally selected "Anderson et al." and the "Fan et al." samples. Since neither of these samples are complete, each of those quasars only occur once in the "high- $z$ sample"; each object was included in the sample which has the better spectrum. The data of the "color-selected quasars" are presented by Fan et al. (1999, 2001b) and Schneider et al. (2001). The photometry of Fan et al. (2001b) and line widths measured in the high $S / N$ spectra of Constantin et al. were used for two objects common to these studies. Five quasars were excluded from the "color-selected sample": the spectra of three of these quasars did not include the $\mathrm{C}$ IV emission line. For another object (J020731.68+010348.9) the poor spectrum quality precludes a FWHM(C IV) measurement. Finally, the spectrum of a fifth object, J225529.09-003433.4, could not be located in any of the references listed or in the SDSS archive. It will be shown in the next section that the "color-selected sample" is statistically similar in the distributions of $L_{\mathrm{bol}}, M_{\mathrm{BH}}$, and $L_{\mathrm{bol}} / L_{\mathrm{Edd}}$ to those of the other high- $z$ quasar samples $(3.5<z \lesssim 5.5)$, as expected if this is a representative subset of $z \approx 4$ quasars.

Pentericci et al. (2002): The authors present nearIR spectra of two of the $z \approx 6$ quasars discovered in SDSS (Fan et al. 2001c), namely SDSSp J103027.10+052455.0 $(z=6.28)$ and SDSSp J130608.26+035626.3 $(z=6.00)$. The C IV line widths are estimated from enlarged versions of the published spectral plots, while the luminosities are adopted from Fan et al. (2001c). The width of C IV measured here for SDSSp J103027.10+052455.0 is consistent with that quoted by Pentericci et al. within the errors. The C IV profile is truncated in the blue-most part of the wing. The FWHM estimate $\left(=7000_{-1000}^{+700} \mathrm{~km} \mathrm{~s}^{-1}\right)$ used here for $M_{\mathrm{BH}}$ was obtained by measuring a reasonable extension of the observed blue profile to the continuum level indicated. The (large) FWHM measurement uncertainty 

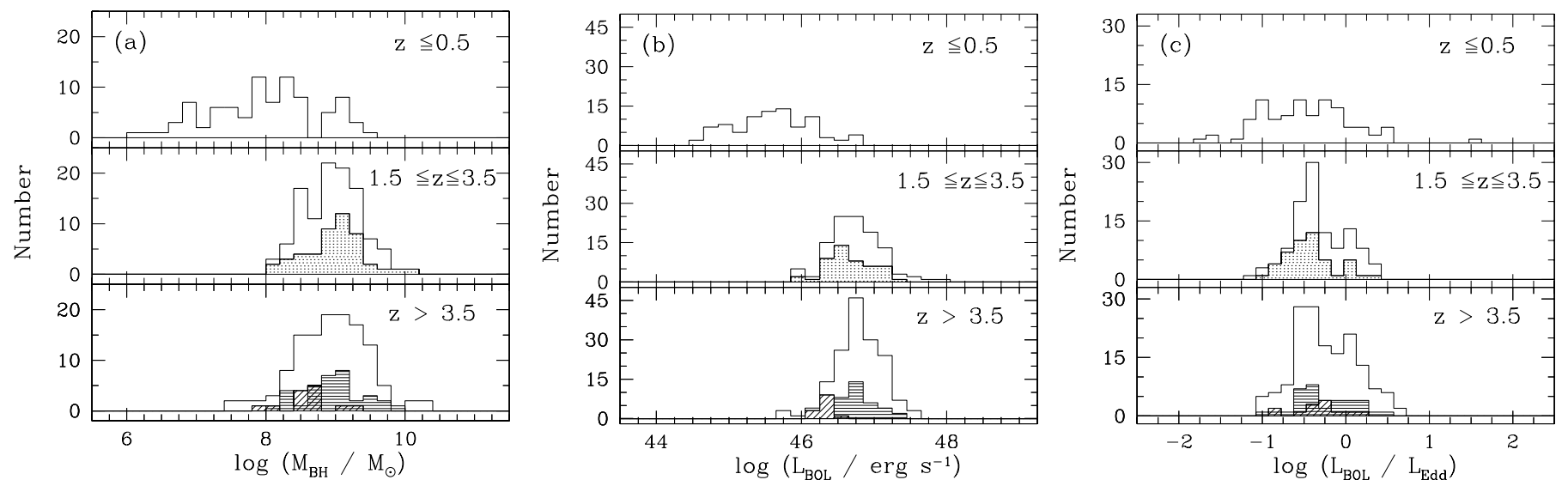

FIG. 1.- Distributions of estimated (a) black-hole mass, $M_{\mathrm{BH}},(b)$ bolometric luminosity, $L_{\mathrm{bol}}$, and (c) Eddington luminosity ratio, $L_{\mathrm{bol}} / L_{\mathrm{Edd}}$ for different redshift bins. The top panel consists of Bright Quasar Survey objects only. The middle panel contains the intermediate$z$ sample; the radio-quiet quasars (RQQs) are shown shaded. The bottom panel unshaded histogram contains all the $(z>3.5)$ quasars in the samples described in $\S 3.3$. The distribution of the faint subsample of Fan et al. (2001b) is high-lighted with diagonal shading. The histogram for the "color-selected sample" of Fan et al. (2001b) is shown with a light horizontal shading for comparison.

adopted is based on a conservative estimate of the continuum uncertainty.

\section{DISTRIBUTIONS OF MASS, LUMINOSITY, AND EDDINGTON RATIO}

\subsection{Number Distributions}

The histograms of masses, bolometric luminosities, and Eddington ratios are shown in Figure 1 for different redshift bins. Figure 2 shows the distributions of emission-line widths with redshift and continuum luminosities.

It is evident from Figure 1 that the intermediate and high-redshift $(z>1.5)$ quasars are all massive $\left(\gtrsim 10^{8} M_{\odot}\right)$ and luminous $\left(\gtrsim 10^{46} \mathrm{ergs}^{-1}\right)$ and have somewhat similar $M_{\mathrm{BH}}$ and $L_{\mathrm{bol}}$ distributions. As discussed in $\S 4.2$ the lower boundaries are due to selection effects. The two radio types of the intermediate-redshift quasars, which are matched in luminosity and redshift, display no significant $M_{\mathrm{BH}}$ difference, in contrast to some studies claiming blackholes of RLQs are more massive (e.g., Laor 2000; McLure $\&$ Dunlop 2001). The radio-loud $z \approx 2$ sample does have a larger number of luminous quasars than the radio-quiet subset (see $\S 3.2$ ). Also, even the quasars in the highredshift $(z \approx 4)$ faint sub-sample of Fan et al. (2001b) are quite massive $\left(\sim 10^{9} M_{\odot}\right)$.

Kolmogorov-Smirnov tests confirm the apparent similarity seen in Figure 1 of the distributions in mass, luminosity, and Eddington ratio for all high-redshift samples (§ 3.3), including the "color-selected sample" of Fan

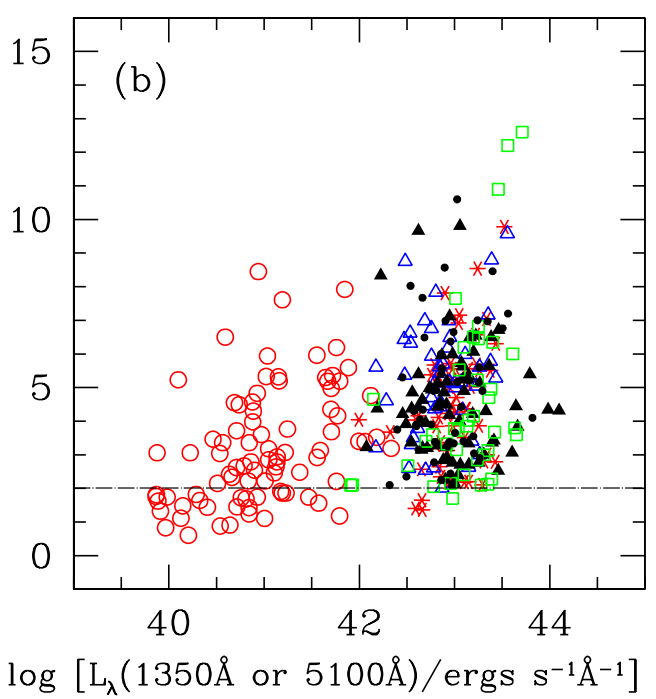

FIG. 2.- Distributions of $\operatorname{FWHM}(\mathrm{H} \beta)$ (for BQS; open circles) and FWHM(C IV) (remaining quasars) with (a) redshift and (b) continuum luminosity, $L_{\lambda}(5100 \AA)$ (for BQS) and $L_{\lambda}\left(1350 \AA\right.$ ) (for the $z>1$ quasars). The horizontal, dashed line marks $\mathrm{FWHM}=2000 \mathrm{~km} \mathrm{~s}{ }^{-1}$, the commonly adopted cutoff for selecting quasars. Samples shown are: BQS (open circles); intermediate redshift radio-quiet (open triangles) and radio-loud (solid triangles) quasars; Constantin et al. sample (open squares); Anderson et al. sample (asterisks); Fan et al. sample and $z \approx 6$ quasars (solid points). 

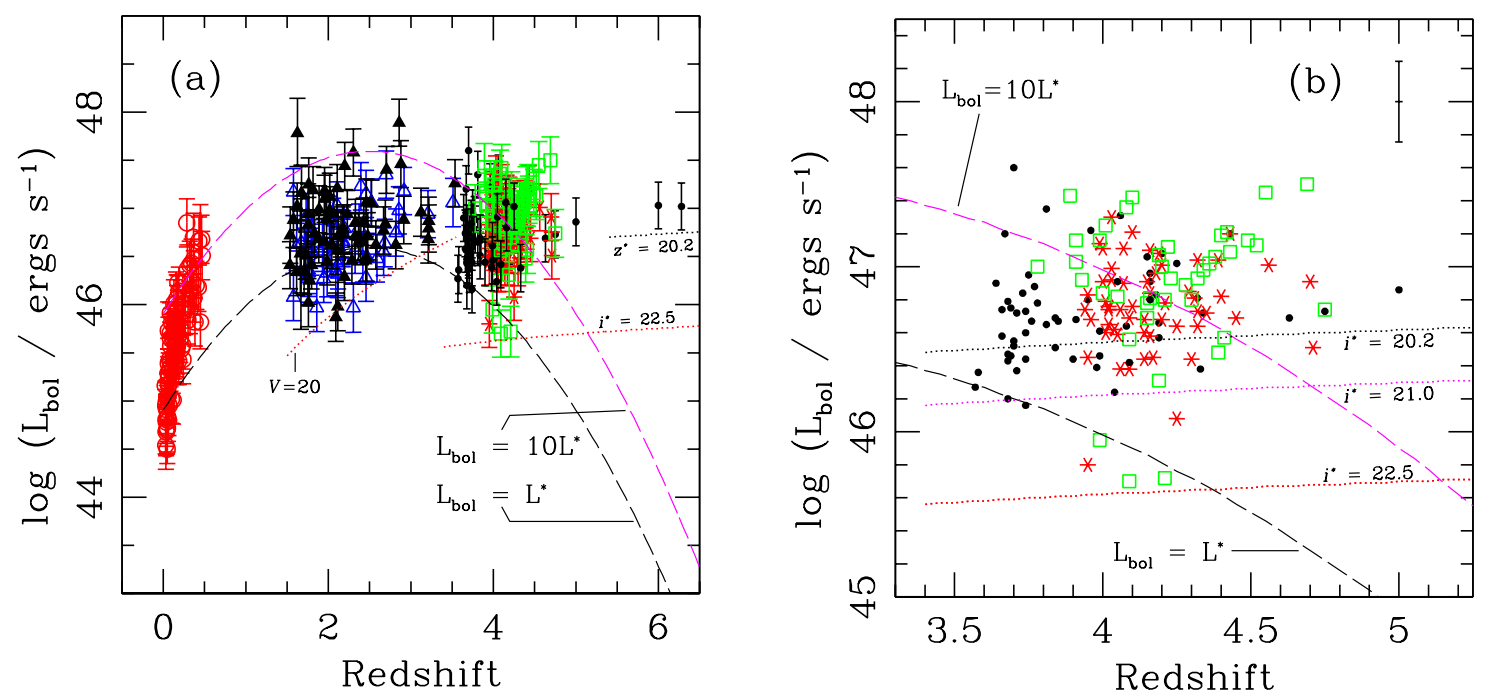

FIG. 3.- Distribution of bolometric luminosities, $L_{\mathrm{bol}}$, as a function of redshift for $(a)$ all the quasar samples considered here and $(b)$ the $z>3.6$ quasar samples only for clarity; error bars are omitted but the average error is shown in upper right corner. See Fig. 2 for symbols. The dashed curves represents the evolution of $L_{\mathrm{bol}}^{*}\left(L_{B}^{*}\right)$ (lower) and $L_{\mathrm{bol}}^{*}\left(10 L_{B}^{*}\right)$ (upper) with redshift. The evolution of $L_{\mathrm{bol}}^{*}$ at $z \gtrsim 2.5$ is an extrapolation of the observed evolution at lower redshift (see text). The dotted curve segments denote limits imposed by sample selection and survey limits. The left panel shows limits for the $z \approx 2$ quasars $(V=20 \mathrm{mag})$, the $z \approx 4$ quasars $\left(i^{*}=22.5 \mathrm{mag}\right)$, and the $z \approx 6$ quasars $\left(z^{*}=20.2 \mathrm{mag}\right)$. The right panel shows the limit of the SDSS in general $\left(i^{*}=22.5 \mathrm{mag}\right)$ and, for reference, the limits of Fan et al. 's "bright" $\left(i^{*}=20.2 \mathrm{mag}\right)$ and "faint" $\left(i^{*}=21 \mathrm{mag}\right)$ samples.

et al. (2001b). The low-redshift quasars of the BQS span a larger range in each of these parameters simply because less-luminous quasars are still bright and easily detectable at low redshift.

Figure 1c suggests that in every redshift range there is a significant number of quasars with $L_{\mathrm{bol}} / L_{\mathrm{Edd}}$ greater than unity. However, given the uncertainties in both $M_{\mathrm{BH}}$ and bolometric corrections, the reality of super-Eddington luminosities is unclear. Nevertheless, barring some large systematic error, all the values of $L_{\mathrm{bol}} / L_{\mathrm{Edd}}$ are high, almost always greater than 0.1 . This is not surprising given that we are sampling the brightest quasars in every redshift bin. Indeed, the lower end of the distribution in $L_{\mathrm{bol}} / L_{\mathrm{Edd}}$ is due to survey flux limits and the apparent upper envelope to the $\mathrm{C}$ IV line width for a given luminosity (Fig $2 \mathrm{~b}$ ). The upper end of the distribution is truncated by the lack of objects with very high luminosity and/or emission lines narrower than about $2000 \mathrm{~km} \mathrm{~s}^{-1}$. Since active nuclei are commonly identified by their broad lines, it is not clear whether the discovery surveys with their selection criteria (and crude spectral resolution in early surveys) would be able to identify quasars with such narrow lines; even the SDSS quasar identification is not well-defined at present (Richards et al. 2001). The upper cutoff in $L_{\mathrm{bol}}$ is however real (see e.g., $\S 4.2$ ).

\subsection{Redshift Distributions}

Figures 3 and 4 show how $L_{\mathrm{bol}}$ and $M_{\mathrm{BH}}$ distribute with redshift. The curve segments are lower limits imposed by the survey limits or sample selection. In Figures 5 and 6 the $M_{\mathrm{BH}}, L_{\mathrm{bol}}$, and $L_{\mathrm{bol}} / L_{\mathrm{Edd}}$ distributions with redshift are shown binned in different ways and with measurement uncertainties omitted for clarity.

The lower envelope of the observed $L_{\mathrm{bol}}$ values is easily explained by the survey or selection flux limits (Fig. 3). The bolometric luminosity corresponding to $L_{B}^{*}$ and $10 L_{B}^{*}$ and their evolutionary tracks [i.e., $L_{\mathrm{bol}}^{*}\left(L_{B}^{*}, z\right)$ and $\left.L_{\mathrm{bol}}^{*}\left(10 L_{B}^{*}, z\right)\right]$ are shown for reference, where $L_{B}^{*}$ is the characteristic (break) luminosity of the quasar luminosity function, $\Phi\left(L_{B}, z\right)$. For simplicity, the parameterization of Boyle et al. (2000) is adopted here, namely:

$$
\Phi\left(L_{B}, z\right)=\frac{\Phi\left(L_{B}^{*}\right)}{\left(L_{B} / L_{B}^{*}\right)^{\alpha}+\left(L_{B} / L_{B}^{*}\right)^{\beta}}
$$

where $L_{\mathrm{B}}$ is the $B$ broad-band luminosity, and $\alpha$ and $\beta$ are the slopes of the faint and bright end of the luminosity function, respectively. The $L_{\mathrm{bol}}^{*}(z)$ values were computed as $10^{1.6} \times L_{\mathrm{B}}^{*}(z)$. The bolometric correction factor to $L_{\mathrm{B}}$ was determined from the updated quasar SED described in $\S 2$. The $L_{\mathrm{B}}^{*}$ and its evolution ${ }^{6}$ was determined for $\sim 6000$ quasars by Boyle et al. (2000) between redshifts zero and 2.3 from the LBQS and 2dF Quasar Redshift Survey data. Boyle et al. extrapolate $L_{\mathrm{B}}^{*}(z)$ to $z>2.3$ based on the fit to the data at lower redshifts. Figure 3 shows that the $3.5 \lesssim z \lesssim 5$ quasars studied here are very luminous: most are well above several $L_{B}^{*}$ (see e.g., Fig. 3b), assuming $L_{\mathrm{bol}}^{*}\left(L_{B}^{*}, z\right)$ can be trusted for short extrapolations (from $z \approx 2.3$ ). The extrapolation to $z \approx 6$ is much more uncertain, but, evidently, the most distant quasars detected are unusually bright. Also, the BQS quasars are clearly among the most luminous quasars at low redshift, as is well known, while the $z \approx 2$ quasars are, on average, $L_{B}^{*}$ quasars or slightly more luminous. A preliminary analysis of the Large Bright Quasar Survey (LBQS) data from Forster et al. (2001) of quasars at $1.3 \lesssim z \lesssim 3.0$ suggests that the brightest of these quasars may exceed $L_{\text {bol }} \approx 10^{47}$

6 The luminosity function determined by Pei (1995) is consistent with that of Boyle et al. (2000) within the parameter uncertainties. 

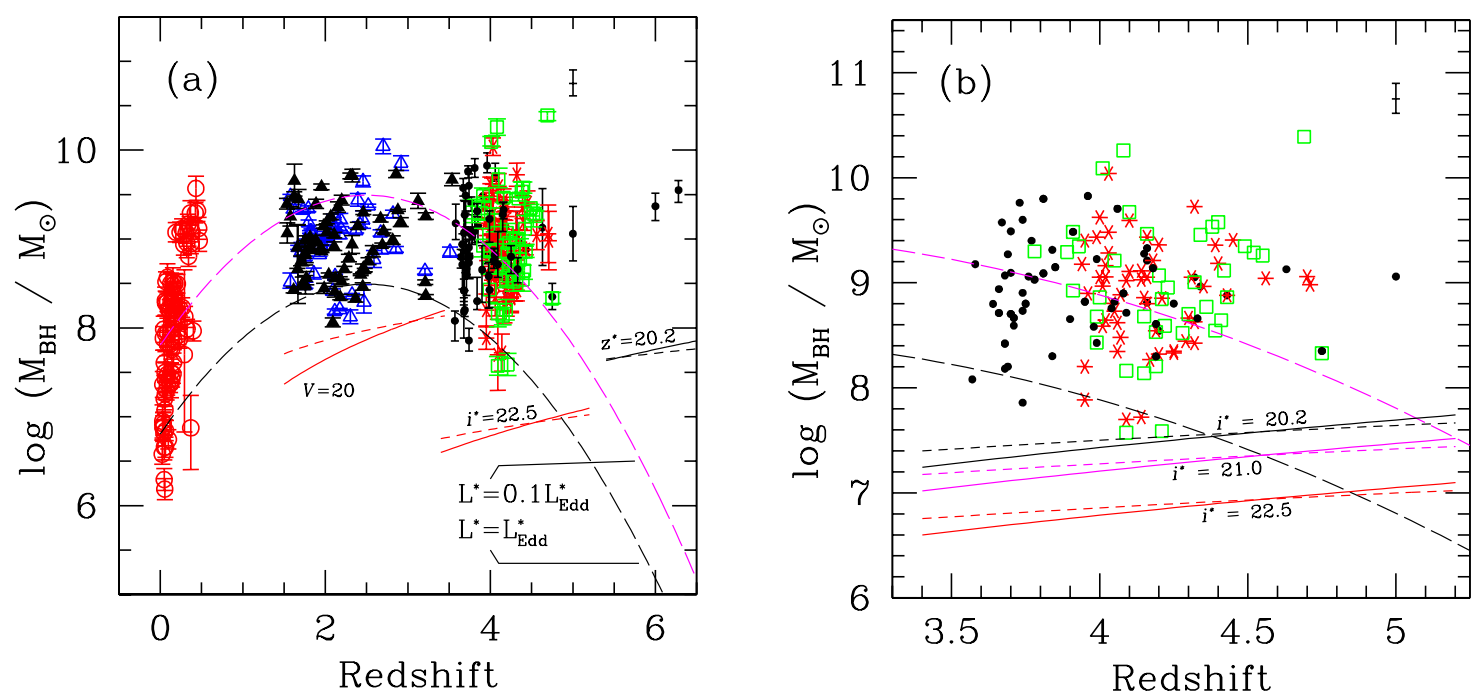

FIG. 4.- Distributions of $M_{\mathrm{BH}}$ with redshift for $(a)$ all samples considered here, and $(b)$ the $3.5 \leq z \leq 5.0$ samples only for clarity; the average error is shown in upper right corner. See Fig. 3 for symbols. The dashed curves show the observed $(z \lesssim 2.5)$ and predicted $(z>2.5)$ evolution of $M_{\mathrm{BH}}^{*}$ determined by assuming $L_{\mathrm{bol}}^{*}=L_{\mathrm{Edd}}^{*}$ (lower) and $L_{\mathrm{bol}}^{*}=0.1 L_{\mathrm{Edd}}^{*}$ (upper), respectively. The line segments denote limits in $M_{\mathrm{BH}}$ imposed by the survey flux limits (see Fig. 3) assuming FWHM(C IV) $=2000 \mathrm{~km} \mathrm{~s}^{-1}\left(1000 \mathrm{~km} \mathrm{~s}^{-1}\right)$ for $z \approx 2(z \gtrsim 4)$ quasars, and adopting the average (dashed segment) and minimum (solid segment) spectral slopes for the quasars at each redshift interval.

$\operatorname{ergs~s}^{-1}$ and $M_{\mathrm{BH}} \approx 10^{10} M_{\odot}$. If indeed so, the relatively lower $L_{\text {bol }}$ values of the intermediate- $z$ sample (see Fig. 3 ) are consistent with the fact that these quasars were intentionally selected not to be the brightest at a given $z$ (Vestergaard 2000).

One possible explanation for the high luminosities is gravitational lensing by foreground galaxies too faint to be detected at present. The lensing fraction is strongly dependent on the slope $(\beta)$ of the bright end of the optical luminosity function at $z>3$ (e.g., Wyithe \& Loeb 2002). Fan et al. (2001a) determined the luminosity function for the well-defined "color-selected sample" of bright quasars at $z \approx 4$ and find a slope of $\beta=2.58$, somewhat flatter than the slope, $\beta=3.43$, of the bright end of the $z \lesssim 2.3$ luminosity function (Boyle et al. 2000). This shows that the $z \approx 4$ "color-selected sample" contains a larger fraction of the most luminous quasars, in accordance with the findings here. More importantly, this bright end slope allows a handle on the lensing fraction at high- $z$, assuming the "color-selected sample" is representative of bright $z \gtrsim 4$ quasars. Wyithe \& Loeb (2002) find a relatively high probability $(P)$ that the luminosity of $z \approx 6$ quasars is enhanced by lensing: $7 \% \lesssim P \lesssim 30 \%$ for $2.58 \leq \beta \leq 3.43$, respectively. The luminosities of the $z \approx 4$ quasars are not expected to be significantly lensed: $4 \% \lesssim P \lesssim 13 \%$. These estimates show that very few of the high- $z$ quasars studied here are likely to be gravitationally lensed. Even if as many as $13 \%$ of the most luminous $z \approx 4$ quasars are lensed, the average $L_{\mathrm{bol}}$ of the remaining quasars is still well above the predicted $L_{\text {bol }}^{*}(z \approx 4):<L_{\text {bol }}>\approx 10^{46.8}$ $\operatorname{ergs~s}^{-1}=9.4 \times<L_{\text {bol }}^{*}(z)>$. Evidently, with the current survey flux limits, these high- $z$ quasar samples only probe the brightest tail of the quasars possibly present at $z \approx 4$. Future surveys need to go much deeper ${ }^{7}$ to properly test whether or not the $z>3$ luminosity function is significantly different than that at lower redshifts.

Figure 4 shows that even the high- $z$ black-hole masses are quite large ${ }^{8}$ and, in particular, do not show a decrease relative to the masses of the intermediate- $z$ quasar sample. However, the masses of the $z \approx 2$ LBQS quasars may reach $10^{10} M_{\odot}$, as noted earlier. Thus, a slight mass decrease may exist at $z>3.5$; the apparent upper envelope in the quasar masses at $4 \lesssim z \lesssim 5$ furthermore suggests this. Also shown in Figure 4 is the relative position of $M_{\mathrm{BH}}^{*}(z)$, determined from the evolution of $L_{\mathrm{bol}}^{*}$ assuming $L_{\mathrm{bol}}^{*} \equiv L_{\mathrm{Edd}}^{*} \propto M_{\mathrm{BH}}^{*}$. Since it is unclear whether all quasars radiate at the Eddington luminosity, the relative location of $M_{\mathrm{BH}}^{*}(z)$, computed assuming $L_{\mathrm{bol}}^{*} \equiv 0.1 L_{\text {Edd }}^{*}$, is also shown for reference. If $L_{\mathrm{bol}}=L_{\mathrm{Edd}}^{*}$ is valid, most of the quasars across the observed redshift range are very massive, reaching $M_{\mathrm{BH}}>10-15 M_{\mathrm{BH}}^{*}$. This is true even at the lowest redshifts $(z<0.6)$. Regardless of the true value of $M_{\mathrm{BH}}^{*}(z \approx 4)$, the $z>3.6$ quasars stand out as being extremely massive. For most quasars, $L_{\mathrm{bol}}=0.1 L_{\text {Edd }}^{*}$ may be reasonable, given previous estimates at lower redshifts (e.g., Wandel et al. 1999; Awaki et al. 2001; Czerny et al. 2001; but see also Collin et al. 2002). In this case, the BQS masses distribute almost evenly about the predicted value of $M_{\mathrm{BH}}^{*}$, with a range of a factor $\sim 10$ in each direction. Also, the values of $M_{\mathrm{BH}}$ for the $z \approx 2$ quasars are mostly in the range $0.1-1 \times M_{\mathrm{BH}}^{*}$. Furthermore, if the $M_{\mathrm{BH}}^{*}(z)$ extrapolation to $z \approx 4$ is reasonable, then the $z>3.6$ quasars have masses in the range $\sim 0.1-10$ $M_{\mathrm{BH}}^{*}$, similar to the BQS. The average observed Edding-

7 An extrapolation of $L_{B}^{*}$ shows that $1.0(0.01) L^{*}$ quasars are expected to have broad-band magnitudes: $i^{*} \approx 25(30)$ mag at $z \approx 5$ and $z^{*} \approx$ 29 (31.5) mag at $z \approx 6$.

8 The mass estimates of the highest redshift $(z=6.41)$ quasar SDSS J114816.64+525150.3 based on both Mg II (Willott et al. 2003a) and C IV line widths (Barth et al. 2003) are in agreement (but note that individual mass estimates based on scaling relationships may be uncertain by a factor 10 (Paper I); the factor 3 uncertainty is valid for statistical samples only). 

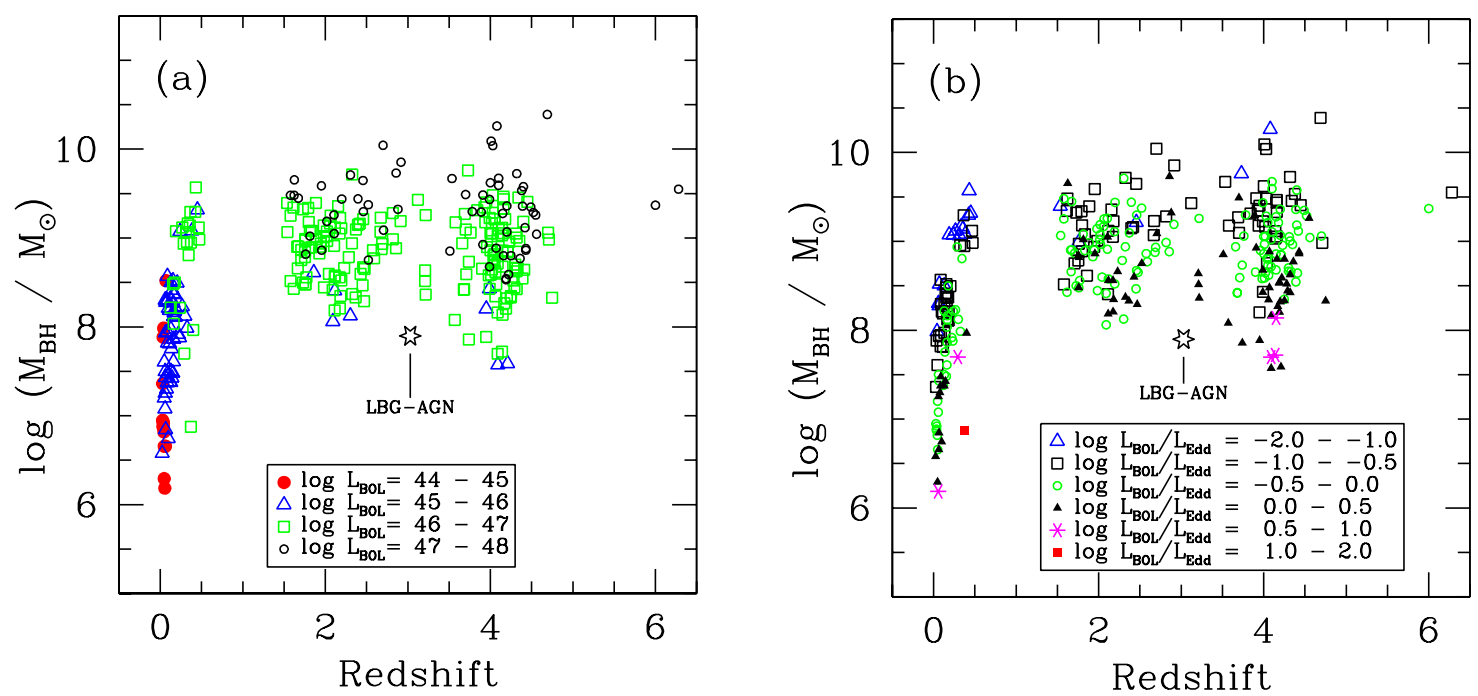

Fig. 5.- Distribution of $M_{\mathrm{BH}}$ with redshift shown binned in $(a) L_{\mathrm{bol}}$ and $(b) L_{\mathrm{bol}} / L_{\mathrm{Edd}}$. Error bars are omitted for clarity. The labeled open star marks the likely position of an "average" broad-lined Lyman-break galaxy AGN (see $§ 5.2$ ).

ton ratio is, however, well above 0.1 (Fig. 1) - possibly closer to a value of 1 for the highest redshift quasars with a typical $L_{\text {bol }} / L_{\text {Edd }}$ value of $0.4-0.5$ at $z>1.5$. Adopting this ratio would make $M_{\mathrm{BH}}^{*}$ fall about halfway between the two $M_{\mathrm{BH}}^{*}(z)$ curves shown in Figure 4, making the $z \approx 2$ quasars $M_{\mathrm{BH}}^{*}$ on average and the $z>3.6$ quasars as massive as $10-15 M_{\mathrm{BH}}^{*}$ or even larger. Note that the absence of much less massive quasars, especially at high- $z$, is mainly due to the selection and survey limits (Figure 4). Also, the high $M_{\mathrm{BH}}$ values are not due to severe overestimates caused by the adopted method to estimate $M_{\mathrm{BH}}(\S 5.1)$.

The very detection of the $z \gtrsim 3.6$ quasars means they are very luminous, as already discussed. But, must they also necessarily be massive? Rejecting the possibility that they are grossly super-Eddington, the obvious possibilities for artificially enhancing their $M_{\mathrm{BH}}$ estimates are (a) gravitational lensing, and (b) relativistic beaming. Gravitational lensing can account for some, but certainly not all quasars (30\% at the very most), as argued above. The high- $z$ quasars are also not likely to be strongly beamed, judging by the similar appearance of their UV spectra to those of low- $z$ AGNs: beamed sources (BL Lac and OVVs) have very weak or absent emission lines (e.g., Peterson 1997). The equivalent width $(E W)$ of the UV lines in the high- $z$ quasars are not much different from those of low- $z$ AGNs. The average $E W(\mathrm{Ly} \alpha+\mathrm{Nv})$ and $E W(\mathrm{C}$ IV $)$ in the Constantin et al. sample are $80 \AA$ and $\sim 40 \AA$, respectively, while Fan et al. (2001b) find a mean $E W(\operatorname{Ly} \alpha+\mathrm{Nv})$ of $69 \AA$ for the "color-selected sample". These compare very well with the typical $E W \mathrm{~s}$ in low$z$ AGNs: $E W(\mathrm{Ly} \alpha+\mathrm{Nv})=75 \AA$ and $E W(\mathrm{C} \mathrm{IV})=35 \AA$ (Peterson 1997). In fact, the $E W$ s at low and high- $z$ are remarkably similar considering more luminous quasars are expected to have lower line $E W$ (the Baldwin effect; e.g., Baldwin 1977). So to conclude, since the $z \gtrsim 3.6$ quasars are rather luminous, they must be massive as well. Additionally, the large emission-line widths (Figure 2) also indicate the black-hole masses are large.
Figure 5 shows that even lower-luminosity $(\sim$ $10^{44} \mathrm{ergs} \mathrm{s}^{-1}$ ) quasars can have relatively high masses, $\approx 10^{8} M_{\odot}$ (the BQS at $z<0.6$ ). The tendency for $L_{\mathrm{bol}} / L_{\mathrm{Edd}}$ to increase with decreasing $M_{\mathrm{BH}}$ is particularly prominent for the $z<0.6$ objects (Fig. $5 \mathrm{~b}$ and $6 \mathrm{~b}$ ). Notably, even the most massive $z>1.5$ quasars also have high Eddington ratios (Fig. 6b). Also, a large fraction of the $M_{\mathrm{BH}} \approx 10^{8}-10^{9} M_{\odot}$ quasars appear to have superEddington luminosities (Figs. 5b and 6b). For the distant $(z>1.5)$ quasars, there is considerable overlap in luminosity for the two highest mass bins (Fig. 6a) and visa-versa (Fig. 5a).

\subsection{Overview of Results}

A number of conclusions can immediately be made based on the distributions described above.

(1) At low redshift $(z<0.6)$, the brightest quasars, represented by the BQS quasars, exhibit a clear upper envelope in $M_{\mathrm{BH}}$ (Fig. 4 and 5) and $L_{\text {bol }}$ (Fig. 3 and 6) which increases with redshift. Although such a sharp upper cutoff is not seen for the more distant quasars there does appear to be a real physical ceiling of $M_{\mathrm{BH}} \approx 10^{10} M_{\odot}$ and $L_{\mathrm{bol}} \approx 10^{48} \mathrm{ergs} \mathrm{s}^{-1}$ at all redshifts. The $L_{\mathrm{bol}} / L_{\mathrm{Edd}}$ ratio evenly covers the same approximate range $(0.1 \lesssim$ $L_{\text {bol }} / L_{\text {Edd }} \lesssim 3$ ) at all redshifts (Figs. 1 and 6 ). The lower envelopes of $M_{\mathrm{BH}}, L_{\mathrm{bol}}$, and $L_{\mathrm{bol}} / L_{\mathrm{Edd}}$ for all the quasars are due to the original discovery surveys being flux limited.

(2) The high- $z$ quasars have relatively high values of $M_{\mathrm{BH}}$ and $L_{\mathrm{bol}}$. The central masses of the highest redshift $(z>3.6)$ quasars do not drop significantly compared to the intermediate redshift $(z \approx 2)$ objects. However, the $z \approx 2$ quasars were not selected to be the brightest, and therefore the most massive, at this redshift (Figs. 1, 3, and 4). A preliminary analysis of the $z>1.3$ LBQS sample suggests that a mild decrease in, at least, $M_{\mathrm{BH}}$ may exist at $z>3.6$, as the brightest $z \approx 2$ LBQS quasars may exceed $M_{\mathrm{BH}} \approx 10^{10} M_{\odot}$ and $L_{\mathrm{bol}} \approx 10^{47} \mathrm{ergs} \mathrm{s}^{-1}$. It is particularly striking that the most distant quasars at $z \approx 6$ have similarly high $M_{\mathrm{BH}} \approx 10^{9} M_{\odot}$ as at lower redshifts. 

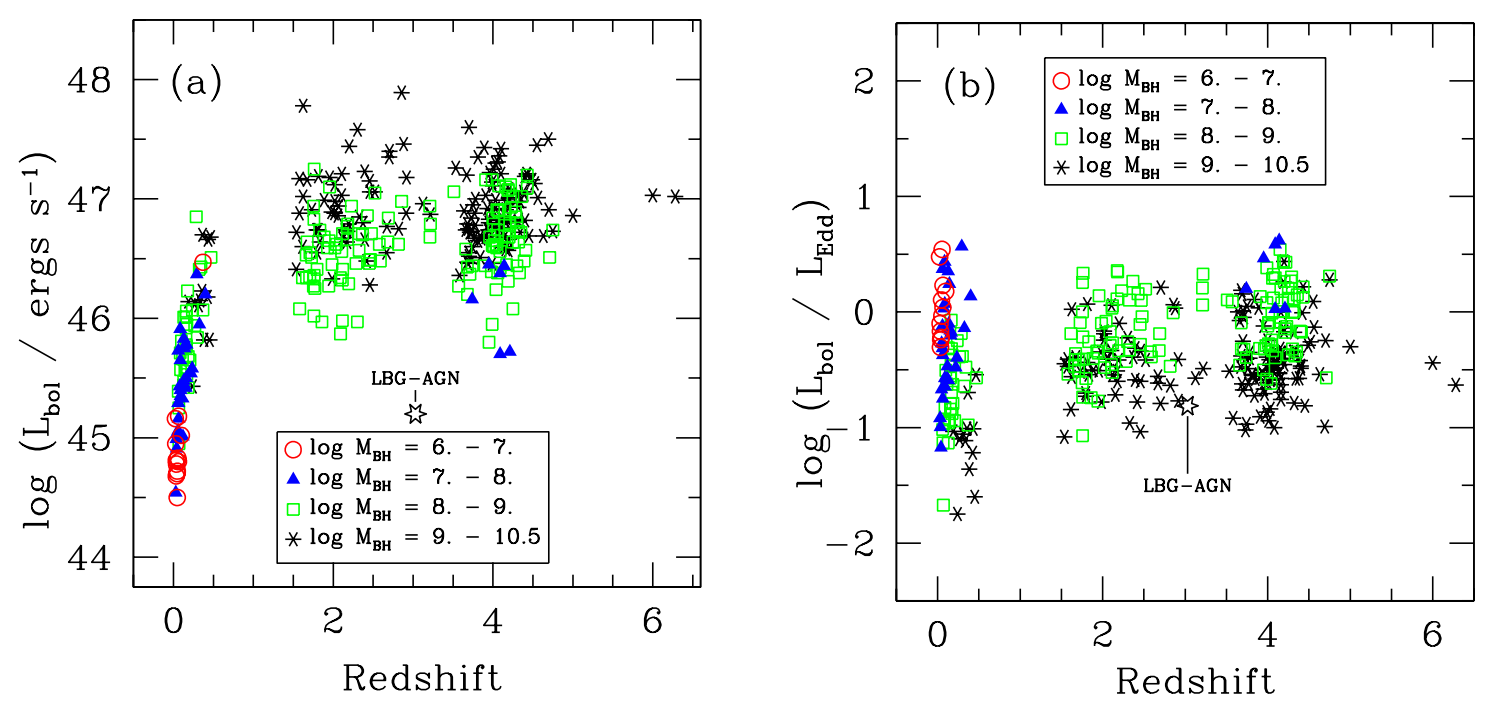

FIG. 6.- Distributions of $(a) L_{\mathrm{bol}}$ and $(b) L_{\mathrm{bol}} / L_{\mathrm{Edd}}$ with redshift shown binned in $M_{\mathrm{BH}}$. Error bars are omitted for clarity. The labeled open star marks the likely position of an "average" broad-lined Lyman-break galaxy AGN (see $\S 5.2$ ).

These quasars do have a high probability $(P \lesssim 30 \%)$ of being gravitationally lensed (Wyithe \& Loeb 2002). But if they are not lensed, the known $z \approx 6$ quasars must be very massive. It has been argued that such massive quasars must necessarily reside in massive dark matter halos and be associated with very rare, high density peaks in the dark matter distribution at that epoch (e.g., Efstathiou \& Rees 1988; Fan et al. 2001c; Volonteri et al. 2002).

(3) Most of the objects have masses above $M_{\mathrm{BH}}^{*}(z)$, even at the lowest redshifts where $L^{*}(z)$ is reliable. Given the mean $L_{\mathrm{bol}} / L_{\mathrm{Edd}}$ at $z>1.5$, it follows that the actual $M_{\mathrm{BH}}^{*}$ in Fig. 4 probably lies between the two dashed curves. This makes the quasar masses of the intermediate- $z$ sample on average $M_{\mathrm{BH}}^{*}$ and makes most of the $z \gtrsim 3.6$ quasars 10 - $15 M_{\mathrm{BH}}^{*}$, if the luminosity function can be reasonably extrapolated to $z \approx 4$.

\section{DISCUSSION}

It was argued in section 2 that black-hole masses of distant AGNs and quasars can be predicted using their broadline widths, their luminosities, and scaling relationships. For a statistical sample of objects this method appears accurate to within a factor of about 3 . When this method is applied to the quasar samples described in $\S 3$ it is concluded that quasars at $4 \lesssim z \lesssim 6$ have black-hole masses of $10^{8}-10^{10} M_{\odot}$, with an average of about $10^{9} M_{\odot}(\S 4)$. In spite the fact that the currently known high- $z$ quasars represent only the upper end of the luminosity function (e.g., Fan et al. 2001a) (and presumably the upper end of the mass function), it is important to appreciate that blackhole masses as large as a few times $10^{9} M_{\odot}$ are capable of forming by $z=6$. Certainly, the high luminosities of these quasars suggest this must be the case (Fan et al. 2001c), but the present results provide evidence of high black-hole masses independently of arguments based only on the Eddington limit.
It is currently unknown how these massive black holes form or on what time scale. As argued by Fan et al. (2001c), it takes $20\left(L_{\mathrm{bol}} / L_{\mathrm{Edd}}\right)^{-1} e$-folding times to grow a $10 M_{\odot}$ black hole with radiative efficiency of $100 \%$ to a mass of $\sim 5 \times 10^{9} M_{\odot}$. As this time scale is within, although close to, the age of the Universe at $z \approx 6$ for a cosmology of $H_{0}=65 \mathrm{~km} \mathrm{~s}^{-1} \mathrm{Mpc}^{-1}, \Omega_{0}=0.35$, and $\Lambda$ $=0.65$, this growth scenario is not ruled out by its time scale.

These conclusions depend on whether or not the scaling relations, defined for nearby AGNs and quasars, can be applied to luminous and very distant quasars. The main justifying reason is that the currently available data at radio, UV, and X-ray wavelengths show no clear evidence that the properties of $z \gtrsim 4$ quasars are any different than those of other luminous quasars known at lower redshift. First, quasar rest-frame UV spectra are very similar at all redshifts up to at least $z \approx 4.5$ (e.g., Constantin et al. 2002; Dietrich et al. 2002a; barring luminosity effects such as the Baldwin effect). Also, as discussed in $\S 4$, the values and distributions of $M_{\mathrm{BH}}, L_{\mathrm{bol}}$, and $L_{\mathrm{bol}} / L_{\mathrm{Edd}}$ are also similar, especially at $z>1.5$. Second, at radio energies, the SDSS $3.6 \lesssim z<5$ quasars exhibit similar properties $(\sim 10 \%$ fractional occurrence and similar radio spectra) as known for $z<3$ quasars (Carilli et al. 2001a; Stern et al. 2000; see Petric et al. 2003 for corroborating results for $z>5$ quasars). Moreover, the X-ray properties of high- $z$ quasars (e.g., the optical-UV to X-ray slope, $\alpha_{\mathrm{OX}}$, and X-ray slope, $\Gamma_{x}$ ) are probably not much different from those of their lower- $z$ cousins. Brandt et al. (2002) and Mathur, Wilkes, \& Ghosh (2002) independently concluded this for the $\alpha_{\mathrm{OX}}$ values of three $z \approx 6$ quasars. Bechtold et al. (2003), on the contrary, argue that $z \gtrsim 4$ quasars have relatively steeper $\alpha_{\text {OX }}$ values $(\gtrsim 1.5$ with an average of 1.6 \pm 0.1 ) based on Chandra data of 17 optically selected, radio-quiet $(3.7 \leq z \leq 6.3)$ quasars, but their $\alpha_{\text {OX }}$ values

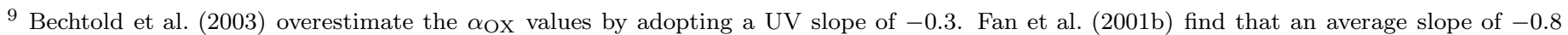

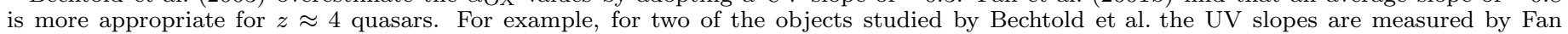

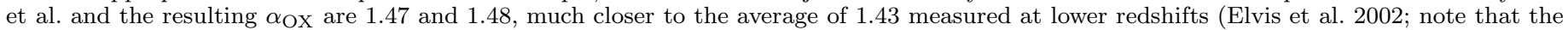


are slightly overestimated ${ }^{9}$. Also, the $\alpha_{\text {OX }}$ slope extends to flatter values $\left(\alpha_{\mathrm{OX}} \approx 1.2\right)$ when $z \approx 4$ quasars from other studies are included (Fig. 1 by Bechtold et al. ), similar to average $\alpha_{\text {OX }}$ values $\left(1.55 \lesssim<\alpha_{\text {OX }}>\lesssim 1.65\right)$ for $z<3$ radio-quiet quasars (Yuan et al. 1998). However, Vignali et al. (2003) also report steeper $\alpha_{\mathrm{OX}}$ values for their $z \approx 4$ quasars, but argue it is a luminosity effect. Nevertheless, Vignali et al. find the X-ray and broad-band spectra similar to those of low- $z$ quasars. Bechtold et al. also determine X-ray slopes of $\Gamma_{x} \approx 2.5(1.5)$ for their $4.0 \leq z \leq 4.5$ $(z \geq 5)$ quasars. These slopes are somewhat flatter than the steepest slopes of 3 to 4 observed for $z \lesssim 2$ ROSAT quasars. However, this apparent flattening of $\Gamma_{x}$ with redshift is most likely a selection effect because different energy ranges of the X-ray spectrum are observed at different redshift, as also suggested by Bechtold et al. (2003). In conclusion, the apparent similar properties across the SED of distant quasars $(z>1.5)$ indicate that the properties of the central engine of the high- $z$ luminous quasars are sufficiently similar that the scaling relationships can used to estimate black-hole masses of these objects. The efficacy of this method is discussed in more detail in $\S 5.1$.

The second key result presented in $\S 4$, is the clear presence of a ceiling to the $L_{\mathrm{bol}}$ and $M_{\mathrm{BH}}$ values at all redshifts. From redshift zero to 0.6 , the maximum values of all three parameters increase to a certain value and then level off at higher redshifts. The upper envelopes in $M_{\mathrm{BH}}$ and $L_{\mathrm{bol}}$ are genuine, since none of the sample selections impose upper limits in the source brightness. Also, there are no apparent selection biases preventing a detection of very broad-lined, bright quasars, with the possible exception of the $z \approx 2$ quasars $(\S 4)$. The apparent lack of very luminous and very massive quasars may be due to either or both of the following explanations. First, very luminous (massive) quasars are expected to be very rare, given the steep decline of the upper end of the luminosity (mass) function. Second, the above-mentioned ceilings may be due to some physical limit to black hole growth. Possible causes for such a maximum sustainable mass and luminosity include: (a) limits in the dark matter gravitational potential, (b) limits in the fuel supply rate, (c) transition of accretion mode or timescale, and (d) changing accretion disk (i.e., emission) properties with black-hole growth (e.g., Caditz, Petrosian, \& Wandel 1991; Yi 1996; Kauffmann \& Haehnelt 2000).

Two recent studies (Bechtold et al. 2003; Netzer 2003) report quasar masses at high- $z(z \approx 4$ and $z \lesssim 3$, respectively) reaching or exceeding $10^{10} M_{\odot}$ in apparent conflict with the above conclusion. However, these $M_{\mathrm{BH}}$ values are not inconsistent with the current analysis once differences in cosmology and statistical uncertainties are taken into account, as explained in the following. Firstly, Netzer (2003) finds a maximum mass of 10.4 dex assuming an $R-L$ slope of 0.7 and $H_{0}=70 \mathrm{~km} \mathrm{~s}^{-1} \mathrm{Mpc}^{-1}, \Omega_{m}=0.3$, and $\Omega_{\Lambda}=0.7$. For this cosmology, his $M_{\mathrm{BH}}$ values are about a factor 2 (or $\lesssim 0.35 \mathrm{dex}$ ) higher than for the cosmology adopted here $\left(H_{0}=75 \mathrm{~km} \mathrm{~s}^{-1} \mathrm{Mpc}^{-1}, \mathrm{q}_{0}=0.5\right.$, and $\Lambda=0$ ). Combining this with the probable uncertainty in his mass values of $\sim 0.6$ dex, given a measurement error of $\lesssim 0.25 \mathrm{dex}$ and a statistical uncertainty in the method of $\sim 0.5$ dex (Paper I), the highest mass values of Netzer's sample and those analyzed here are statistically consistent. An important point to keep in mind is that individual $M_{\mathrm{BH}}$ values can be in error by as much as a factor 10 . The fraction $(\lesssim 9 \%)$ of quasars in Netzer's sample with $M_{\mathrm{BH}}$ $>10^{10} M_{\odot}$ is also within the expected fraction $(\sim 10 \%)$ of objects for which the $M_{\mathrm{BH}}$ values may be off by 1 dex or more (Paper I). Secondly, the maximum $M_{\mathrm{BH}}$ value $(\sim 10.7 \mathrm{dex})$ reported by Bechtold et al. (2003) for $H_{0}=$ $50 \mathrm{~km} \mathrm{~s}^{-1} \mathrm{Mpc}^{-1}, \mathrm{q}_{0}=0.5$, and $\Lambda=0$, corresponds to $\sim 10.45$ dex in the current cosmology. Although, Bechtold et al. do not quote their errors, the uncertainties in their $M_{\mathrm{BH}}$ values are presumably as large as or even larger ${ }^{10}$ than those of Netzer (2003), and, hence, their maximum $M_{\mathrm{BH}}$ value is similarly consistent with the upper boundary $\leq 10^{10} M_{\odot}$, seen in the current study, to within the errors. Netzer (2003) also claims that $M_{\mathrm{BH}} \gtrsim 10^{10} M_{\odot}$ is inconsistent with the locally defined $M_{\mathrm{BH}}-\sigma$ relationship ( $\sigma$ is the stellar velocity dispersion in the host galaxy bulge), since the implied $\sigma \approx 700 \mathrm{~km} \mathrm{~s}^{-1}$ is not observed locally. As Netzer notes, either (1) the $M_{\mathrm{BH}}$ values are overestimated, because, for example, the $R-L$ relationship does not extrapolate to high- $z$ quasars, or (2) the $M_{\mathrm{BH}}-\sigma$ relationship changes with redshift. It is argued in $\S 5.1$ that the $R-L$ relationship is not likely to be misleading and that the scaling relationships used here yield reasonable $M_{\mathrm{BH}}$ estimates to within a factor $\lesssim 4$. Furthermore, the lack of local black-holes with masses $\sim 10^{10} M_{\odot}$ does not mean that the large $M_{\mathrm{BH}}$ values inferred from the scaling relationships are strongly overestimated. The reason is that in addition to a possible evolution of the $M_{\mathrm{BH}}-\sigma$ relationship and the statistical uncertainty in the $M_{\mathrm{BH}}$ estimate, the lack of local black-holes with masses $\sim 10^{10} M_{\odot}$ can possibly be explained by the relatively small volume probed in the local Universe: for the comoving space density of luminous, massive $\left(\gtrsim 5 \times 10^{9} M_{\odot}\right)$ quasars at $z \approx 4$ of $\lesssim 12.5 \mathrm{Gpc}^{-3}$ (Vestergaard \& Osmer, in preparation), a volume approximately 20 times larger than that currently probed locally $(\sim 100 \mathrm{Mpc})$ is needed to detect one such massive quiescent black-hole.

\subsection{Reliability of the Mass Estimates for High Redshift Quasars}

It is a serious concern whether the scaling relationship, defined for a representative sample of nearby AGNs and quasars, can rightfully be applied to luminous, distant quasars. Specifically, can extension of this relationship cause the masses of the distant quasars $\left(\sim 10^{9} M_{\odot}\right)$ to be severely overestimated? Netzer (2003) also points out that the high masses he finds at $z \lesssim 3$ appear inconsistent with the local $M_{\mathrm{BH}}-\sigma$ relationship. In this section, the efficacy of the scaling relationships for luminous, distant quasars is discussed. It is argued that application of the scaling relationships to such quasars is valid, owing mainly to the similarity of quasar spectra across the known redshift and luminosity ranges. In particular, the scaling relation is

radio-quiet quasars observed with $R O S A T$ have $\left\langle\alpha_{\mathrm{OX}}\right\rangle=1.55$; Yuan et al. 1998). It may thus not be the norm that high-redshift quasars have very steep optical-X-ray slopes; but see Vignali et al. 2003).

10 This is owing to their crude correction of their $\operatorname{FWHM}(\mathrm{C}$ IV $)$ values to $\operatorname{FWHM}(\mathrm{H} \beta)$, so to apply the optical scaling relationship of $\operatorname{Kaspi}$ et al. (2000) to obtain central masses, and their adoption of rather flat UV slopes $(-0.3)$ relative to the steep slopes $(-0.8)$ measured by Fan et al. (2001b) for their representative $z \approx 4$ quasars (see footnote above). 
unlikely to be inaccurate by a factor $\gtrsim 4$ in the mass.

The mass estimates will obviously be misleading if the assumptions made when extending the scaling relationships beyond the nearby AGNs break down. The two assumptions for $M_{\mathrm{BH}} \propto \mathrm{FWHM}(\mathrm{C} \mathrm{IV})^{2} \lambda L_{\lambda}^{0.7}(1350 \AA)$ are: (1) that the size, $R_{\mathrm{BLR}}$, of the (C IV) emitting region in the broad line region - i.e., its distance from the continuum source - can be estimated reliably from the continuum luminosity, $L_{\lambda}(1350 \AA)$, and (2) that the line width is a reasonable proxy for the gravity-dominated velocity dispersion of the line-emitting gas. The critical issues are therefore: (a) whether or not the $R-L$ relationship is valid at higher redshift and for more luminous quasars than the sample for which it was defined, and (b) whether or not the C IV emission gas is virialized or, for example, the $\mathrm{C}$ IV profile has strong blue asymmetries which may be a sign of BLR outflows as suggested for the narrow-line Seyfert 1 (NLS1) galaxies (e.g., Leighly 2000). Each issue is addressed in turn below.

First, the $R-L$ relationship is not likely to be much different for the more luminous and more distant AGNs. The reasons are twofold: (1) the $R-L$ relationship is not extrapolated very far in luminosity: for the highest $\lambda L_{\lambda}(1350 \AA)$ values of $47.4 \mathrm{dex}$, the $R-L$ relationship is extrapolated by less than $1.5 \mathrm{dex}$, a small fraction of the luminosity range of $\gtrsim 4 \mathrm{dex}$, over which the $R-L$ relationship is defined $\left(10^{42} \lesssim \lambda L_{\lambda}(5100 \AA) / \mathrm{ergs} \mathrm{s}^{-1} \lesssim 10^{46}\right)$, and (2) AGN and quasar spectra look very similar at all redshifts and luminosities considered here, i.e., line flux ratios, line equivalent widths, and SEDs are all similar (e.g., Elvis et al. 1994; Kuhn et al. 2001; Constantin et al. 2002; Dietrich et al. 2002a, 2002b; see also § 2). Specifically, if the $R-L$ relationship does not apply to luminous, distant quasars, the spectra of these objects would look somewhat different than they do. This can be quantified by photoionization modeling. In essence, if the scaling relationship (eq. 4) results in a mass overestimate of, for example, a factor 10 for a quasar with $L_{\mathrm{bol}} \approx 10^{47} \mathrm{ergs} \mathrm{s}^{-1}$, the virial theorem shows that the $\operatorname{Ly} \alpha$ and $\mathrm{C}$ IV emission lines are emitted so close $(\lesssim 33$ light-days $)$ to the ionizing source that these lines are produced very inefficiently, and, in particular, C III] emission cannot be generated. But, the presence of $\mathrm{C}$ III] in all spectra of quasars (at least up to $z \approx 4.3$; Dietrich et al. 2002a) confirms the existence of low-density BLR gas subject to a lower ionizing flux from which Ly $\alpha$ and CIV are also much more efficiently emitted ${ }^{11}$ : this gas will necessarily be located at a greater distance from the ionizing source, close to the distance inferred from the luminosity.

Second, there is no evidence that the kinematics of the BLR in high- $z$ AGNs are not dominated by gravity. This conclusion is based on the following 3 arguments.

1. Most, if not all, of the BLR gas ${ }^{12}$ is in virial motion around the central source, including the highionization UV lines: all the AGNs with suitable multiple emission line measurements demonstrate a robust virial relationship between their line widths and variability time lags (Peterson \& Wandel 1999, 2000; Onken \& Peterson 2002); while only four AGNs are testable so far, the general similarity of AGN spectra (i.e., line shape, line widths, and variability properties) argues that this relationship extends to most AGNs.

2. The distribution of C IV line widths in spectra of distant, luminous quasars is similar to that for nearby AGNs (typical values $\sim 4500 \mathrm{~km} \mathrm{~s}^{-1}$ with a range from $2000-10000 \mathrm{~km} \mathrm{~s}^{-1}$ in FWHM), including those for which the scaling relations are calibrated. Also, the C IV widths of the nearby BQS quasars are consistent with their $\mathrm{H} \beta$ line widths, assuming virial BLR kinematics and that $\mathrm{H} \beta$ is emitted twice as far from the central source as C IV, as established for three of the four AGNs discussed in item 1 (see also Korista et al. 1995; see comment below on the one exception). Furthermore, the similar FWHM(C IV) distributions, where most are narrower than $\sim 8000 \mathrm{~km} \mathrm{~s}^{-1}$ (e.g., Figure 2), argue that the unknown, but possible, contribution to single-epoch line widths from non-variable optically thin emission (Shields, Ferland, \& Peterson 1995; Peterson 1997) is highly unlikely to make FWHM(CIV) in error by a factor $\gtrsim 2$; such an error translates to a mass uncertainty of a factor $\lesssim 4$, comparable to the statistical uncertainty $(\S 2)$.

3. Outflow of the BLR line-emitting gas is not important, at least for the quasars considered here. This is supported by two facts: (i) none of the CIV profiles of the $z>1.5$ quasars $(\S 3.2, \S 3.3)$, and most quasars in general, clearly ${ }^{13}$ resemble the broad, triangular, blue asymmetric C IV profiles of the high luminosity NLS1s; those C IV profiles have been argued to signify strongly outflowing high-ionization line-emitting gas (e.g., Leighly 2000 ), as NLS1s are very luminous for their $M_{\mathrm{BH}}$, and (ii) while a small fraction $(\leqslant 20 \%)$ of the CIV profiles in the high- $z$ sample $(\S 3.3)$ show weak, blue-ward asymmetries (mostly suspected due to broad, blended NIV] $\lambda 1486$ ), those quasars do not have much larger masses and luminosities than the remaining quasars: a conservative estimate is an 0.2 dex increase on average, dominated by the lower-quality spectra of the Anderson et al. sample; the 'asymmetric subset' of the Constantin et al. and Fan et al. quasars exhibit average deviations of \pm 0.1 dex. Also, the C IV profiles of the $z \approx 2$ quasars are not typically asymmetric, and any asymmetries are restricted to the very base of the profile and do not affect FWHM. Moreover, the strongest asymmetries do not extend blueward (Vestergaard 2000; Vestergaard, Wilkes, \& Barthel 2000).

In conclusion, blue asymmetries and UV outflows are generally not a concern for the $M_{\mathrm{BH}}$ estimates of quasars.

\footnotetext{
11 These conclusions are based on the line emission resulting from gas with a range of column densities subject to the ionizing flux from a source with $L_{\mathrm{bol}} \approx 10^{47} \mathrm{ergs} \mathrm{s}^{-1}$ as quantified by the photoionization grids of Korista et al. (1997).

12 This has so far been established for the following emission lines: Si IV $\lambda 1400$, C IV $\lambda 1549$, He II $\lambda 1640$, C III] $\lambda 1908$, H $\beta \lambda 4861$, He II $\lambda 4686$ (see e.g., Peterson \& Wandel 1999).

13 Some spectra are noisy and the reality of such a prominent triangular line shape is difficult to discern.
} 
Nonetheless, extreme care should be exercised when using the C IV line width to estimate the mass when signs of significant outflows are present. For this reason, mass estimates based on C IV are not well suited for the most luminous NLS1s.

It is important to emphasize that although all AGNs so far tested show the BLR is virialized (i.e., the virial product $R v^{2}$ is constant for all emission lines measured), for one object (3C 390.3) C IV is narrower than $\mathrm{H} \beta$ and has a larger lag in contrast to the other three objects. This behavior is not understood, but 3C 390.3 may have an unusual BLR structure as it is the only broad-line radiogalaxy with monitoring data and it, moreover, has doublepeaked Balmer lines (Peterson \& Wandel 2000). Nevertheless, it is very important for the general applicability of the scaling relations that future studies address this issue.

\subsection{Do Black Holes "Mature" Before Their Host Galaxies Do?}

Given that black holes with large masses appear as early as $z \approx 6$ and given the intimate connection between the formation of galaxy bulges and their central, supermassive black holes inferred from the $M_{\mathrm{BH}}-\sigma$ relationship, the question naturally arises whether or not this close connection also exists at high redshift. Specifically, do these "mature" black holes (i.e., those that have reached, say, $\sim 10^{8}-10^{9} M_{\odot}$ ) also reside in "mature" host galaxies (i.e., virialized systems with the majority of their stars already formed) with aged (>1 Gyr) stellar populations? Among the currently available data on high- $z$ AGN host galaxies, there is considerable circumstantial evidence that massive active black holes may, in fact, reside in galaxies that are not fully relaxed, virialized systems and where many of the stars are yet to be formed. This is based on (a) the inferred high star-formation rates (SFRs) from the existing large masses of cool dust, (b) the presence of significant amounts of molecular gas in some sources, (c) the presence of large-scale young starbursts in at least one well-studied $z \approx 4$ radio-galaxy with a large dust mass, (d) the strikingly different morphology of host galaxies of high- $z$ radioquiet quasars and radio galaxies compared to their lower- $z$ cousins, and (e) the presence of massive, active black-holes in small, young galaxies at $z \gtrsim 3$. Each issue is discussed in turn below.

\subsubsection{The Evidence}

Massive Star Formation in High- $z$ Quasars High$z$ AGN host galaxies are apparently experiencing massive, large-scale star formation: the far-IR/sub-mm SEDs of $z \approx 4$ quasars (e.g., Benford et al. 1999; Carilli, Menten, \& Yun 1999; Carilli et al. 2001a) are similar to those of ultra-luminous IR galaxies (ULIRGs) and starburst galaxies (e.g., Downes \& Solomon 1998; Genzel et al. 1998; Rowan-Robinson 2000; Klaas et al. 2001). ULIRGs are dominated by extreme rates of star formation $\left(\approx 1000 M_{\odot}\right.$ $\mathrm{yr}^{-1}$ ) that yield unique far-IR/sub-mm SEDs, which are independent of whether or not a central AGN is present (e.g., Downes \& Solomon 1998; Klaas et al. 2001). The rather cool $(T<100 \mathrm{~K}$; typically $\sim 40-50 \mathrm{~K})$ starburstheated dust dominates the far-IR emission (e.g., Rowan-
Robinson 2000; Dunne et al. 2000; Klaas et al. 2001), but AGN-heated dust $(T>100 \mathrm{~K})$ emits in the near-IR, shortward of $\sim 60 \mu \mathrm{m}$ (e.g., Wilkes et al. 1999b; van Bemmel \& Dullemond 2003; Figure 8a by Farrah et al. 2003). Large amounts $\left(\gtrsim 10^{8} M_{\odot}\right)$ of cool $(\sim 40-50 \mathrm{~K})$ dust are commonly inferred ${ }^{14}$ from the restframe far-IR/sub-mm emission from $z \approx 4$ quasars (e.g., Benford et al. 1999; Carilli et al. 2001a; Omont et al. 2001; Priddey \& McMahon 2001; Isaak et al. 2002). Large dust masses are often taken to imply high SFRs, $\sim 1000-2000 M_{\odot} \mathrm{yr}^{-1}$ (Benford et al. 1999; Carilli et al. 1999, 2000, 2001b, 2003); such high SFRs are even inferred for the undetected sources (Isaak et al. 2002). Also, non-thermal radio emission contributes little to the shape of the $\mathrm{cm}$ to sub-mm SED, confirming its origin in massive star formation (Carilli et al. 2000, 2001a, 2001b; Yun et al. 2000).

The far-IR properties of the luminous $z \approx 4$ SDSS quasars studied here appear very similar to those of other $z \approx 4$ quasars with far-IR data and to far-IR selected AGNs. Most of them have radio to far-IR SEDs consistent with starburst heating of large dust masses (Carilli et al. 2001a), and the inferred dust masses $\left(M_{\text {dust }}\right)$ and SFRs are similar to other $z \approx 4$ quasars: Figure 7 shows that for the subset of 30 quasars with $M_{\mathrm{BH}}$ and $L_{\mathrm{bol}}$ estimates, the 13 quasars detected at $1.2 \mathrm{~mm}$ all have $M_{\mathrm{BH}}$ $\gtrsim 10^{9} M_{\odot}$. Even for the undetected quasars, the upper limits cannot rule out the existence of SFRs of order 300 $-700 M_{\odot} \mathrm{yr}^{-1}$.

This line of argument is not without caveats. For example, it can be argued (e.g., Sanders et al. 1989; Kuraszkiewicz et al. 2003) that the IR SEDs of nearby quasars can be explained purely by AGN heating, although this explanation is not universally accepted (e.g., Farrah et al. 2003; Klaas et al. 2001). Nevertheless, no correlation is observed between quasar optical-UV luminosity and far-IR luminosity, as otherwise expected if AGN heating dominates the cooler dust (e.g., McMahon et al. 1999; Omont et al. 2001; Isaak et al. 2002; Priddey et al. 2003). Also, Archibald et al. (2002) argue that a high dust mass may indicate a galaxy is in a late starforming phase $(\gtrsim 75 \%$ complete), as discussed below.

Molecular Gas. Huge amounts of cold molecular gas $\left(>10^{11} M_{\odot}\right)$ are present in $z>3$ far-IR luminous sources. This is (a) determined from CO emission line measurements (Ohta et al. 1996; Omont et al. 1996; Guilloteau et al. 1999; Carilli et al. 1999, 2002a, 2002b; Papadopoulos et al. 2000), and (b) inferred from the dust masses and typical dust-to-gas ratios obtained from low- $z$ starbursts (e.g., Sanders, Scoville, \& Soifer 1991; Dunne et al. 2000). Such vast gas reservoirs show that large amounts of stars are yet to be formed (e.g., Solomon et al. 1997; Downes \& Solomon 1998), and are evidence for ongoing massive star formation, especially when combined with large masses of cool dust (e.g., Ohta et al. 1996; Ivison et al. 1998; Frayer et al. 1999; Papadopoulos et al. 2000; Carilli et al. 2002a, 2002c). In some cases, the cold gas clearly constitutes a significant fraction of the dynamical mass in the system, emphasizing the rather young nature of the galaxy (e.g., Ohta et al. 1996; Guilloteau et al. 1999; Solomon

\footnotetext{
14 The inferred dust masses are, in fact, lower limits as cold dust $(T<30 \mathrm{~K})$ is a poor emitter (e.g., Omont et al. 2001; Chapman et al. 2003);
} there is probably a factor $\sim 3$ more gas (e.g., Dunne et al. 2000). 
et al. 1997; Downes \& Solomon 1998; Tacconi et al. 1999). For some high- $z$ sources, the sub-mm emission is (a) clearly separated (tens of kpc) from the AGN nucleus, and/or is (b) distinctly extended (e.g., Papadopoulos et al. 2000; Omont et al. 2001; Isaak et al. 2002; Carilli et al. 2001a, $2002 \mathrm{~b}, 2003)$. This is strongly suggestive of galacticscale starforming regions and renders an AGN origin less likely (Carilli et al. 2003). Furthermore, when CO observations of high- $z$ sources are conclusive, the high masses of cold gas inferred from dust mass estimates are confirmed (Hughes et al. 1993; Ohta et al. 1996; Omont et al. 1996; Papadopoulos et al. 2000). Non-detections of CO line emission, however, do not necessarily rule out a young starburst interpretation because the observations are still not very sensitive ${ }^{15}$.

Young Stellar Populations. For one far-IR/sub-mm luminous high- $z$ AGN, the $z \approx 3.8$ radio galaxy $4 \mathrm{C} 41.17$, stellar populations synthesis modeling has established the presence of large-scale young starbursts with ages in the range $0.01 \mathrm{Gyr}$ to $0.4 \mathrm{Gyr}$ with a most probable age of 0.07 Gyr (Dey et al. 1997; Dey 1999). This is also important because it confirms the existence of starburst activity, expected based on the strong far-IR emission alone. Although it is difficult to generalize based on one object, it is pertinent to emphasize that such analysis is very difficult to perform for most high- $z$ AGNs, given the strong nuclear glare in quasars and the faintness of galaxies at high- $z$. Nevertheless, it is important for future studies to establish whether or not high- $z$ far-IR luminous AGNs in general have young starburst activity.

Archibald et al. (2002) infer starburst ages of 0.5 $0.8 \mathrm{Gyr}$ for $4 \mathrm{C} 41.17$ from a monochromatic luminosity ratio and their models of dust production and depletion with time in a young galaxy, and conclude the galaxy is in a late starforming phase. These ages are clearly at odds with the results of the more sophisticated age determination discussed above. Further developments in this area seem necessary to resolve these apparent inconsistencies. More importantly, the measured dust mass of $4 \mathrm{C} 41.17$ is also consistent with a starburst age in the range 0.075 Gyr to 0.095 Gyr (Fig. 1 by Archibald et al. 2002), in good agreement with the results of Dey (1999). In conclusion, while dust contents alone is an inaccurate measure of starburst ages, large dust masses are not uniquely tied to $\gtrsim 1 \mathrm{Gyr}$ old galaxies but also appear in galaxies with starbursts as young as $0.04 \mathrm{Gyr}$ (for $M_{\text {dust }} \gtrsim 10^{8} M_{\odot} ;$ Fig. 1 by Archibald et al. 2002).

It is therefore an intriguing possibility that some high- $z$ AGN host galaxies may have rather young stellar populations. However, a comparison with the restframe IR emission is necessary to determine the extent to which these young stars dominate the total stellar mass in place at that epoch. The observed apparent extension of the $K-z$ relation, defined for $z<1.6$ radio galaxies (Lily \& Longair 1984), to $z \gtrsim 3$ radio galaxies and quasars is sometimes used to argue for somewhat old stellar populations in their host galaxies. However, it is important to appreciate that the increasing scatter in the observed relationship at $z>2$ is also consistent with galaxies comprising mainly young stellar populations forming at $4 \lesssim z \lesssim 5$ (De Breuck et al. 2002): the stellar mass in the host galaxies (quantified by the $K$-band magnitude) is too low in many cases to place all high- $z$ radio-galaxies on the $K-z$ relation with insignificant scatter. Also, it has been argued that the nature of proto-galaxies or young, less massive galaxies may also conspire to fall along the $K-z$ relation at $z \gtrsim 3$ (Eales \& Rawlings 1993; Willott et al. 2003b). Hence, it seems at least plausible that a good fraction of high- $z$ host galaxies may be relatively young, even if this interpretation is not universally accepted.

Host Galaxy Morphology While quasar host galaxies appear fully assembled by $z \approx 1$, AGN host galaxy properties become increasingly more difficult to quantify at $z>1$ (Kukula et al. 2001). Practically, radio galaxies are the only AGNs we can currently study beyond $z \approx 3$, owing to the lack of nuclear glare that affects optical observations of quasar hosts. Radio-galaxy hosts do not appear fully assembled and dynamically relaxed. Specifically, in contrast to their lower- $z$ cousins with mostly single, smooth components, $z>3$ radio galaxies display very clumpy structure: multiple, small star-forming components are embedded in large-scale $(\sim 50 \mathrm{kpc})$ faint emission, indicative of galactic systems in early stages of assembly (e.g., Graham et al. 1994; van Breugel et al. 1998; Pentericci et al. 1999, 2001; Papadopoulos et al. 2000). Optically obscured $z>2$ radio galaxies also appear to be in an early stage of formation (Reuland et al. 2003).

For high- $z$ radio-quiet quasar hosts, the best-quality imaging data available are likely those from NICMOS $H S T$ studies of $2 \lesssim z \lesssim 3$ sources (Ridgway et al. 2001; Kukula et al. 2001). In contrast to radio-galaxy hosts, these are relatively structureless, single components. Also, at $2 \lesssim z \lesssim 3$, radio-quiet hosts are often more compact (scale-lengths $\lesssim 4 \mathrm{kpc}$ and $\sim 2.3 \mathrm{kpc}$ on average; Ridgway et al. 2001) than (a) radio-loud hosts at that epoch (scalelengths $\sim 11-18 \mathrm{kpc}$; e.g., Best et al. 1998; Kukula et al. 2001; Ridgway et al. 2001), and than (b) quasar hosts at $z \approx 0.2$ (mean scale-lengths of $\sim 8.2 \mathrm{kpc}$; Bahcall et al. 1997; McLure et al. 1999). Moreover, the hosts of $z \approx 2$ radio-quiet quasars are apparently rather young: Ridgway et al. find the $2 \lesssim z \lesssim 3\left(M_{B} \approx-24 \mathrm{mag}\right)$ radio-quiet quasar hosts contain only $\sim 10 \%-20 \%$ of their final stellar mass. A caveat is that the Ridgway et al. results may not reflect the properties of the $2-3$ magnitude more luminous radio-quiet quasars observed at $z \approx 4$, but deep imaging of $z>3$ radio-quiet quasar hosts is currently unavailable.

Can AGNs Occur in Young Galaxies? Probably the most convincing evidence that relatively young galaxies at $z \gtrsim 3$ are capable of hosting massive, active black holes comes, somewhat surprisingly, from the distant, smallscaled, young, star-forming Lyman-break galaxies (LBGs; e.g., Steidel et al. 1996, 1999). The presence of an active black hole can be inferred for a tiny fraction $(\sim 1 \%)$ of the $\sim 1000$ LBGs detected in a survey at $z \approx 3$ (Stei-

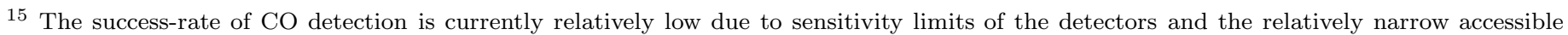

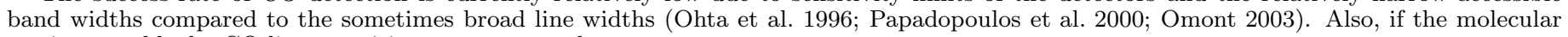
gas is too cold, the CO line transitions are very weak. 


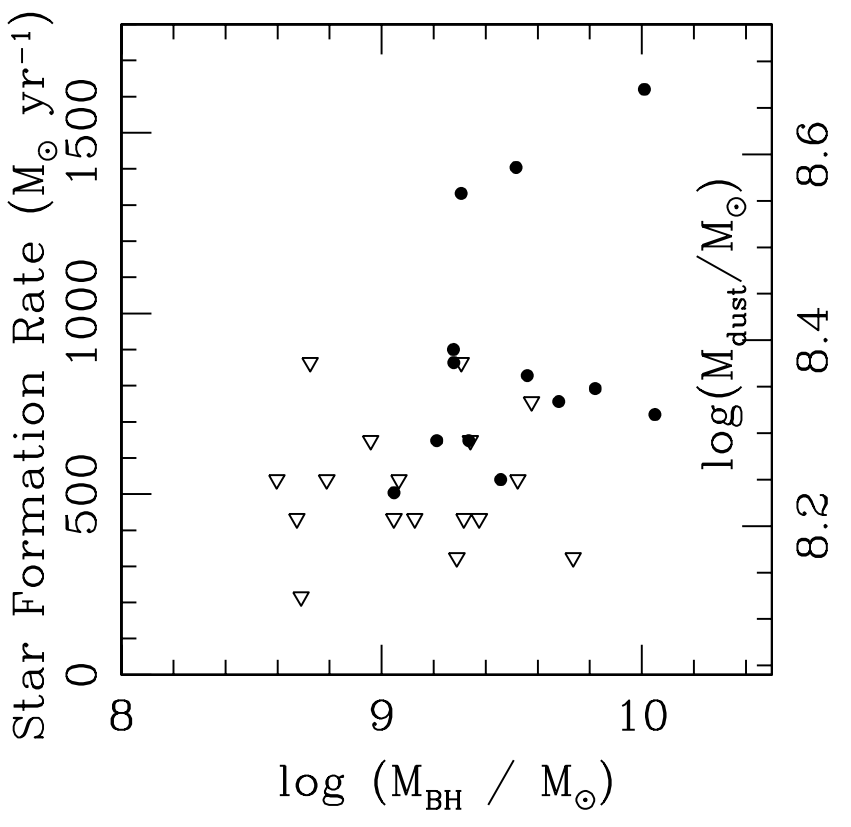

del et al. 2002) by the presence of broad emission lines in their rest-UV spectra. Specifically, the average spectrum of these broad-lined LBGs (Steidel et al. 2002) suggests that the mass of the black hole is typically rather high $\left(\gtrsim 10^{8} M_{\odot}\right)$ : Table 1 lists estimates of the average values of $M_{\mathrm{BH}}, L_{\mathrm{bol}}$, and $L_{\mathrm{bol}} / L_{\mathrm{Edd}}$ for different cosmologies and assumed dust extinction factors. Based on the uncorrected observed fluxes, the broad-lined LBGs have masses $M_{\mathrm{BH}}$ $\approx 10^{8} M_{\odot}$, lower than the higher luminosity quasars at any redshift beyond 1.5 (Figure 5), and they are about an order of magnitude less luminous (Figure 6). In spite of the large uncertainties, the inferred average $M_{\mathrm{BH}}$ value is intriguingly large considering (a) the relatively faint nature of both the nuclear and host galaxy emission, (b) the starformation activity in the host galaxy (Steidel et al. 1996), and (c) the low stellar mass in the galaxies at $z \approx 3$ : they typically only contain $\sim 10 \%-30 \%$ of their expected final stellar mass (Ridgway et al. 2001; Papovich et al. 2001). This suggests that the massive central black hole in the LBGs formed early and fast compared to the underlying stellar component.

A possible caveat is the uncertainty as to whether or not these faint LBG-type AGNs have similar SEDs to the bright quasars, on which both the $M_{\mathrm{BH}}$ and, especially, $L_{\text {bol }}$ estimates are based. But among the lowerluminosity AGNs in the nearby Universe, only the faint, very low-luminosity LINERs display dramatically different SEDs (Ho 1999), mainly missing the "big blue bump" emission from the accretion disk. Seyfert galaxies, with which the LBGs with broad high-ionization lines may be most similar, display only minor SED differences in the optical-UV region compared to the more luminous quasars (e.g., Sanders et al. 1989; Elvis et al. 1994). Moreover, the relatively large $\mathrm{CIV}$ line widths $\left(4670 \pm 500 \mathrm{kms}^{-1}\right.$; very similar to the mean FWHM(C IV) of the luminous $z \approx 4$ quasars) and line equivalent widths (see Figure 1 by Steidel et al. ) support a high central mass (as argued in $\S 4.2$ ), in spite the faint nature of the galaxies. Therefore, the derived mass and luminosity values are crude, but probably
FIG. 7.- Distribution of inferred star formation rate (SFR) and mass of dust with estimated black-hole mass for a subset of the $z \approx 4$ SDSS quasars analyzed here. The far-IR emission emitted by the cool dust was observed by Carilli et al. (2001a). Detections at $\lambda_{\text {obs }}=1.2 \mathrm{~mm}$ are shown as solid circles, while the open triangles denote $3 \sigma$ upper limits. The dust masses and SFRs are here computed using the prescription of Omont et al. (2001). Specifically, SFR $\approx 360 \delta_{\mathrm{MF}} \delta_{\mathrm{SF}}\left(S_{250 \mathrm{GHz}} / m J y\right) M_{\odot} \mathrm{yr}^{-1}$, where $\delta_{\mathrm{SF}}$ is the ratio between the starburst's total luminosity and the farIR luminosity, and $\delta_{\mathrm{MF}}$ is "a function of the present mass composition of the stellar population" (see Omont et al. for explanation) and has values in the range $0.8 \lesssim \delta_{\mathrm{MF}} \lesssim 2$. Since starburst heating dominates the far-IR, $\delta_{\mathrm{SF}} \gtrsim 70 \%$ is assumed, and thus $0.6 \lesssim \delta_{\mathrm{MF}} \delta_{\mathrm{SF}} \lesssim 1.4$; a fair estimate is $\delta_{\mathrm{MF}} \delta_{\mathrm{SF}} \approx 1$ yielding a lower limit on the SFR.

not too unrealistic.

\subsubsection{Discussion and Summary}

Although largely circumstantial, the evidence presented above suggests that the $z \gtrsim 3$ massive, active black holes typically reside in relatively young (possibly dynamically unrelaxed) galaxies in their major star-forming phase. The implication is thus that the black hole either formed long before the stars in the galaxy or sometime during the first star-formation phase and thereafter grew so fast relative to the stellar mass that the nucleus shined long before the galaxy was fully formed. Rix et al. (2001) and Omont et al. (2001) independently reached similar conclusions based on different arguments. Rix et al. find $z \approx 2$ radio-quiet quasars to have either higher nuclear luminosities or fainter host galaxies than lower- $z$ AGNs. They argue that the most plausible explanation is that the ratio of $M_{\mathrm{BH}}$ to the stellar mass increases with redshift. Omont et al. (2001) use millimeter data on $z \approx 4$ quasars to argue for a higher black-hole growth rate relative to the surrounding bulge stellar component in the host galaxy at early epochs, consistent with the current study and that of Rix et al. Such an evolutionary scenario is consistent with some hierarchical models (e.g., Haehnelt \& Rees 1993). Also, Silk \& Rees (1998) suggest that early blackhole growth might in fact help regulate star formation in the host galaxy and its evolution. The overall conclusion reached here is thus that black holes likely reach maturity before the stellar populations in their host galaxies.

Archibald et al. (2002) claim that quasar activity is delayed $0.5-0.7 \mathrm{Gyr}$ from the onset of star formation, during which $\gtrsim 75 \%$ of the stars are formed, in apparent contradiction to the results presented above. However, Archibald et al. assume that black holes grow by accretion with a radiative efficiency $(\eta)$ of $10 \%$, while the value of $\eta$ and its constancy with time (or redshift) are highly uncertain. A lower radiative efficiency allows faster black-hole growth, and indeed, some recent estimates imply $\eta \approx 0.03$ (Schirber \& Bullock 2003). Furthermore, one can equally 
TABLE 1

ESTIMATED $^{a}$ MASS, LUMINOSITY, AND EDDINGTON RATIO OF THE AVERAGE BROAD-LINED AGN AMONG LYMAN-BREAK GALAXIES.

\begin{tabular}{|c|c|c|c|c|c|}
\hline $\begin{array}{l}\text { Extinction } \\
\text { Factor }\end{array}$ & $\begin{array}{c}H_{0} \\
\left(\mathrm{~km} \mathrm{~s}^{-1}\right)\end{array}$ & $\begin{array}{l}\log L_{\nu}(1350 \AA) \\
\left(\operatorname{erg~s}^{-1} \mathrm{~Hz}^{-1}\right)\end{array}$ & $\begin{array}{c}\log M_{\mathrm{BH}} \\
\left(M_{\odot}\right)\end{array}$ & $\begin{array}{l}\log L_{\mathrm{bol}} \\
\left(\operatorname{erg~s}^{-1}\right)\end{array}$ & $L_{\mathrm{bol}} / L_{\mathrm{Edd}}$ \\
\hline Applied & $\left.\mathrm{Mpc}^{-1}\right)$ & & & & \\
\hline None & 50 & $\frac{(\mathrm{J})}{29.54 \pm 0.02}$ & $\frac{(4)}{8.2 \pm 0.1}$ & $\frac{(3)}{45.56+0.2}$ & $\frac{\text { (b) }}{0.20 \pm 0.10}$ \\
\hline None & 75 & $29.19 \pm 0.02$ & $7.9 \pm 0.1$ & $45.20 \pm 0.2$ & $0.15 \pm 0.08$ \\
\hline 50 & 50 & $31.24 \pm 0.02$ & $9.4 \pm 0.1$ & $47.26 \pm 0.2$ & $0.64 \pm 0.32$ \\
\hline 50 & 75 & $30.89 \pm 0.02$ & $9.1 \pm 0.1$ & $46.90 \pm 0.2$ & $0.50 \pm 0.25$ \\
\hline
\end{tabular}

a These estimates and measurement uncertainties are based on the composite spectrum of Steidel et al. (2002). While it is based on non-spectrophotometric data, it should after all be representative of the mean brightness level of these AGNs (C. Steidel, 2002, private communication), sufficient for the discussion here. Specifically, the typical C IV line width is measured (from the published plot and similarly to the other data analyzed here; $\S 3$ ) to be $4670 \pm 500 \mathrm{~km} \mathrm{~s}^{-1}$, and the $1350 \AA$ flux is $(2.01 \pm 0.09) \mu \mathrm{Jy}$. Since the composite spectrum shows evidence of some dust reddening, the $M_{\mathrm{BH}}, L_{\mathrm{bol}}$, and $L_{\mathrm{bol}} / L_{\mathrm{Edd}}$ values were also computed after making a correction for dust extinction. Steidel et al. (2002) estimate an extinction factor of $\sim 50$ for their narrow-lined AGNs, but do not quote such a factor for the broad-lined AGNs among their LBGs. Therefore, the estimate for the narrow-lined AGNs were adopted here in attempts to bracket reality somewhat. In addition to the listed values of $H_{0}, q_{0}=0.5$ is assumed.

well expect black-hole formation and early mass growth to be very fast, as expected for the first generation of massive stars (e.g., Abel, Bryan, Norman 2002). Silk \& Rees (1998) find it unlikely that the first black holes formed with $M_{\mathrm{BH}}<10^{6} M_{\odot}$; in that case, it would take $\leq 0.2 \mathrm{Gyr}$ to generate a $10^{8} M_{\odot}$ black hole from a $10^{6} M_{\odot}$ seed accreting with $\eta \lesssim 10 \%$, consistent with quasar activity existing in very young galaxies, as it takes $0.3 \mathrm{Gyr}$ to form $50 \%$ of the stars in the galaxy (Archibald et al. 2002).

It is pertinent to re-emphasize that the presence of young starbursts in high- $z$ quasars cannot be inferred from their high dust content alone: a young evolving galaxy maintains a large dust mass $\left(\gtrsim 10^{8} M_{\odot}\right)$ from $0.04 \mathrm{Gyr}$ to $1.5 \mathrm{Gyr}$ after the initial starburst (e.g., Archibald et al. 2002). But the combination of high masses of cool dust and cold (molecular) gas is much more convincing. Therefore, it is important for future investigations to study the cold gas contents in high- $z$ AGN host galaxies to test whether or not they are in the process of building up the majority of their stars. Furthermore, better coverage of the radio through near-IR SEDs of high- $z$ quasars is required to constrain the relative contributions of AGN and starburst heated dust for more accurate estimates of SFRs.

\section{CONCLUSIONS}

The main results of this work can be summarized as follows:

(1) It is confirmed that the high- $z$ quasars have central masses of at least a few times $10^{9} M_{\odot}$ as previously estimated from their luminosities (Fan et al. 2001c). The masses, bolometric luminosities, and Eddington ratios of the $z \gtrsim 3.6$ quasars are very similar to those of their lowerredshift cousins. Relative to the evolved $L_{\mathrm{bol}}$ and $M_{\mathrm{BH}}$ the $z \approx 4$ quasars are much more luminous and more massive than the typical quasars at $z<3.5$. This assumes that the luminosity function, based on the Large Bright Quasar Survey and the 2-Degree Field Quasar Redshift Survey, and its extension to $z \approx 4$ is reasonably reliable $(\S 4.2)$.

(2) The scaling laws from which the black-hole mass is estimated are fully applicable to distant luminous quasars. This is argued based on the similarity of quasar and AGN spectral properties (line widths, line equivalent widths, line ratios, SEDs) at low and high redshift. In particular, photoionization models confirm that if the radius - luminosity relationship does not extend to high- $z$ quasars, their spectra would look different than they do. Mass estimates, based on scaling relationships, are not likely inaccurate by a factor greater than $\sim 3$ to $\sim 4(\S 5.1)$ when applied to statistically significant samples.

(3) There is a real ceiling to the $M_{\mathrm{BH}}$ and $L_{\mathrm{bol}}$ values at all redshifts: $M_{\mathrm{BH}}<10^{10} M_{\odot}$ and $L_{\mathrm{bol}}<10^{48} \mathrm{ergs} \mathrm{s}^{-1}$. These upper limits are consistent with the recent studies of Netzer (2003) and Bechtold et al. (2003) to within the uncertainties and differences in adopted cosmologies. Moreover, the large $M_{\mathrm{BH}}$ values at high- $z$ are not inconsistent with local $M_{\mathrm{BH}}$ values given the much smaller volume probed locally $(\S 5)$.

(4) Black holes reach maturity (i.e., reach masses $M_{\mathrm{BH}}$ $\approx 10^{8}-10^{9} M_{\odot}$ ) before their host galaxies: AGN host galaxies at $z \gtrsim 3$ do not typically appear fully assembled, dynamically relaxed, and/or the majority of their stars are not formed or are somewhat evolved ( $>1 \mathrm{Gyr})$. Since the evidence for relatively young quasar-host galaxies at $z>3$ is mostly indirect, this interpretation needs to be tested further. For example, complete coverage of the radio to IR SEDs of large representative samples of $z>3$ quasars will help provide improved constraints on the relative starburst and AGN contributions to the far-IR emission. Detailed studies confirming the correlation between 
large dust masses, large reservoirs of cold molecular gas, and high star-formation rates in larger quasar samples are also required. No doubt, the combination of SIRTF and the planned FIRST/Herschel and ALMA telescopes will play an important part in shedding light on these issues.

Thanks are due to Anca Constantin, Fred Hamann, Joe Shields, and Xiaohui Fan for providing the digital spectra of their $z \approx 4$ quasars, Martin Elvis for providing a digital form of the average quasar SEDs, Dave Sanders for providing his $L_{\mathrm{bol}}$ measurements of the Palomar-Green quasars, and Adam Steed for updating the radio-quiet SED. Luis Ho, Kirk Korista, and Pat Osmer are all thanked for discussions or helpful comments. Special thanks go to Brad
Peterson for countless extensive and very valuable discussions, and for comments on the manuscript. I am also thankful that Luis Ho, Pat Osmer, and Brad Peterson were brave enough to read and comment on an early, larger version of the manuscript; their input was very helpful and much appreciated. The author gratefully acknowledges financial support from the Columbus Fellowship. Part of the observations reported here were obtained at the MMT Observatory, a joint facility of the Smithsonian Institution and the University of Arizona. This research has made use of the NASA/IPAC Extragalactic Database (NED) which is operated by the Jet Propulsion Laboratory, California Institute of Technology, under contract with the National Aeronautics and Space Administration.

\section{REFERENCES}

Abel, T., Bryan, G. L., \& Norman, M. L. 2002, Science, 295, 93

Anderson, S. F. et al 2001, AJ, 122, 503

Archibald, E. N., Dunlop, J. S., Jimenez, R., Friaça, A. C. S., McLure, R. J., \& Hughes, D. H. 2002, MNRAS, 336, 353

Awaki, H., Terashima, Y., Hayashida, K., \& Sakano, M. 2001, PASJ, 53,647

Bahcall, J. N., Kirhakos, S., Saxe, D. H., \& Schneider, D. P. 1997, ApJ, 479, 642

Baldwin, J. A. 1977, ApJ, 214, 679

Barth, A. J., Martini, P., Nelson, C. H., Ho, L. C. 2003, preprint (astro-ph/0308005)

Barthel, P. D., Miley, G. K., Schilizzi, R. T, Lonsdale, C. J. 1988, A\&AS, 73, 515

Barthel, P. D., Tytler, D. R., Thomson, B. 1990, A\&AS, 82, 339

Bechtold, J. et al. 2003, ApJ, 588, 119

Benford, D. J., Cox, P., Omont, A., Phillips, T. G., \& McMahon, R. G. 1999, ApJ, 518, L65

Benson, A. J., Lacey, C. G., Baugh, C. M., Cole, S., \& Frenk, C. S. 2002, MNRAS, 333, 156

Best, P. N., Longair, M. S., \& Röttgering, H. J. A. 1998, MNRAS, 295,549

Boroson, T. A., \& Green, R. F. 1992, ApJS, 80, 109

Boroson, T. A. \& Oke, J. B. 1982, Nature, 296, 397

Boroson, T. A., Oke, J. B., \& Green, R. F. 1982, ApJ, 263, 32

Boyle, B. J., Shanks, T., Croom, S. M., Smith, R. J., Miller, L., Loaring, N., \& Heymans, C. 2000, MNRAS, 317, 1014

Brandt, W. N. et al. 2002, ApJ, 569, L5

Caditz, D. M., Petrosian, V., \& Wandel, A. 1991, ApJ, 372, L63

Cardelli, J. A., Clayton, G. C., Mathis, J. S., 1989, ApJ, 345, 245

Carilli, C. L., Menten, K. M., \& Yun, M. S. 1999, ApJ, 521, L25

Carilli, C. L. et al. 2000, ApJ, 533, L13

Carilli, C. L. et al. 2001a, ApJ, 555, 625

Carilli, C. L., Bertoldi, F., Omont, A., Cox, P., McMahon, R. G., \& Isaak, K. G. 2001b, AJ, 122, 1679

Carilli, C. L. et al. 2002a, AJ, 123, 1838

Carilli, C. L. et al. 2002b, ApJ, 575, 145

Carilli, C. L., Bertoldi, F., Menten, K., \& Owen, F. 2002c, in Galaxy Evolution, Theory and Observations, in press

Carilli, C. L., Lewis, G. F., Djorgovski, S. G., Mahabal, A., Cox, P., Bertoldi, F., \& Omont, A. 2003, Science, 300, 773

Cattaneo, A., Haehnelt, M. G., \& Rees, M. J. 1999, MNRAS, 308, 77

Chapman, S. C., Blain, A. W., Ivison, R. J., Smail, I. 2003, in Star formation through time, preprint (astro-ph/0304236)

Colina, L. et al. 2001, ApJ, 563, 546

Collin, S., Boisson, C., Mouchet, M., Dumont, A.-M., Coupé, S., Porquet, D., \& Rokaki, E. 2002, A\&A, 388, 771

Constantin, A., Shields, J. C., Hamann, F., Foltz, C. B., \& Chaffee, F. H. 2002, ApJ, 565, 50

Czerny, B., Nikołajuk, M., Piasecki, M., \& Kuraszkiewicz, J. 2001, MNRAS, 325, 865

De Breuck, C., van Breugel, W., Stanford, S. A., Röttgering, H., Miley, G., \&'Stern, D. 2002, AJ, 123, 637

Dey, A. 1999, in The Most Distant Radio Galaxies, ed. H. J. A. Röttgering, P. N. Best, \& M. D. Lehnert, (Amsterdam: Royal Netherlands Academy of Arts and Sciences), 19

Dey, A., van Breugel, W., Vacca, W. D., \& Antonucci, R. 1997, ApJ, 490, 698

Dietrich, M., Appenzeller, I., Vestergaard, M., \& Wagner, S. J. 2002b, ApJ, 564, 581
Dietrich, M., Hamann, F., Shields, J.C., Constantin, A., Vestergaard, M., Chaffee, F., Foltz, C.B., Junkkarinen, V.T. 2002a, ApJ, 581, 912

Di Matteo, T., Croft, R. A. C., Springel, V., Hernquist, L. 2003, ApJ, 593,56

Downes, D. \& Solomon, P. M. 1998, ApJ, 507, 615

Dunne, L., Eales, S., Edmunds, M., Ivison, R., Alexander, P., \& Clements, D. L. 2000, MNRAS, 315, 115

Eales, S. A. \& Rawlings, S. 1993, ApJ, 411, 67

Efstathiou, G. \& Rees, M. J. 1988, MNRAS, 230, 5P

Elvis, M. et al. 1994, ApJS, 95, 1

Elvis, M., Risaliti, G., \& Zamorani, G. 2002, ApJ, 565, L75

Fan, X. et al. 1999, AJ, 118, 1

Fan, X. et al. 2000, AJ, 119, 1

Fan, X. et al. 2001a, AJ, 121, 54

Fan, X. et al. 2001b, AJ, 121, 31

Fan, X. et al. 2001c, AJ, 122, 2833

Fan, X., Narayanan, V. K., Strauss, M. A., White, R. L., Becker, R. H., Pentericci, L., \& Rix, H. 2002, AJ, 123, 1247

Farrah, D., Afonso, J., Efstathiou, A., Rowan-Robinson, M., Fox, M., \& Clements, D. 2003, MNRAS, 343, 585

Ferrarese, L., 2002, ApJ, 578, 90

Ferrarese, L., \& Merritt, D. 2000, 539, L9

Ferrarese, L., Pogge, R. W., Peterson, B. M., Merritt, D., Wandel, A., \& Joseph, C. L. 2001, ApJ, 555, L79

Forster, K., Green, P. J., Aldcroft, T. L., Vestergaard, M., Foltz, C. B., \& Hewett, P. C. 2001, ApJS, 134, 35

Francis, P. J., Hewett, P. C., Foltz, C. B., Chaffee, F. H., Weymann, R. J., \& Morris, S. L. 1991, ApJ, 373, 465

Frayer, D. T. et al. 1999, ApJ, 514, L13

Gebhardt, K., et al. 2000a, ApJ, 539, L13

Gebhardt, K., et al. 2000b, ApJ, 543, L5

Genzel, R. et al. 1998, ApJ, 498, 579

Graham, J. R. et al. 1994, ApJ, 420, L5

Guilloteau, S., Omont, A., Cox, P., McMahon, R. G., \& Petitjean, P. 1999, A\&A, 349, 363

Haehnelt, M. G., \& Kauffmann, G. 2000, MNRAS, 318, L35

Haehnelt, M. G. \& Rees, M. J. 1993, MNRAS, 263, 168

Haehnelt, M. Natarajan, P., \& Rees, M. J. 1998, MNRAS, 300, 817

Haiman, Z. \& Loeb, A. 1998, ApJ, 503, 505

Haiman, Z. \& Loeb, A. 2001, ApJ, 552, 459

Haiman, Z. , Madau, P., \& Loeb, A. 1999, ApJ, 514, 535

Hartwick, F. D. A. \& Schade, D. 1990, ARA\&A, 28, 437

Hasinger, G. 2002, in New Visions of the X-ray Universe in the XMMNewton and Chandra Era, Ed. F. Jansen (ESTEC: ESA SP-488), XX (astro-ph/0202430)

Hewitt, A. \& Burbidge, G. 1993, ApJS, 87, 451

Ho, L. C. 1999, ApJ, 516, 672

Hook, I. M., McMahon, R. G., Shaver, P. A., \& Snellen, I. A. G. 2002, A\&A, 391, 509

Huchra, J. \& Burg, R. 1992, ApJ, 393, 90

Hughes, D. H., Robson, E. I., Dunlop, J. S., \& Gear, W. K. 1993, MNRAS, 263, 607

Isaak, K. G., Priddey, R. S., McMahon, R. G., Omont, A., Peroux, C., Sharp, R. G., \& Withington, S. 2002, MNRAS, 329, 149

Ivison, R. J. et al. 1998, ApJ, 494, 211

Kaspi, S., Smith, P. S., Netzer, H., Maoz, D., Jannuzi, B. T., \& Giveon, U. 2000, ApJ, 533, 631

Kauffmann, G., \& Haehnelt, M. 2000, MNRAS, 311, 576

Kellerman, K. I., Sramek, R., Schmidt, M., Shaffer, D. B., \& Green, R. 1989, AJ, 98, 1195

Kennefick, J. D., et al. 1995a, AJ, 110, 78 
Kennefick, J. D., Djorgovski, S. G., \& de Carvalho, R. R. 1995b, AJ, 110,2553

Kennefick, J. D., Djorgovski, S. G., \& Meylan, G. 1996, AJ, 111, 1816

Klaas, U, et al. 2001, A\&A, 379, 823

Korista, K. T., et al. 1995, ApJS, 97, 285

Korista, K., Baldwin, J., Ferland, G., \& Verner, D. 1997, ApJS, 108, 401

Kuhn, O., Elvis, M., Bechtold, J., \& Elston, R. 2001, ApJS, 136, 225.

Kukula, M. J., Dunlop, J. S., McLure, R. J., Miller, L., Percival, W. J., Baum, S. A., \& O'Dea, C. P. 2001, MNRAS, 326, 1533

Kuraszkiewicz, J. K. et al. 2003, ApJ, 590, 128

Lanzetta, K.-M., Chen, H.-W., Pascarelle, S., \& Yahata, N. 1999, in The Birth of Galaxies, ed. B. Guiderdoni et al. (Gif-sur-Yvette, Editions Frontiéres), in press (astro-ph/9809294)

Laor, A. 2000, ApJ, 543, L111

Laor, A., Bahcall, J. N., Jannuzi, B. T., Schneider, D. P., Green, R. F., \& Hartig, G. F. 1994, ApJ, 420, 110

Leighly, K. M. 2000, New Astronomy Review, 44, 395

Lilly, S. J. \& Longair, M. S. 1984, MNRAS, 211, 833

Lonsdale, C. J., Smith, H. E., \& Lonsdale, C. J. 1995, ApJ, 438, 632

Mathur, S., Wilkes, B. J., \& Ghosh, H. 2002, ApJ, 570, L5

McLure, R. J. \& Dunlop, J. S. 2001, MNRAS, 327, 199

McLure, R. J., Kukula, M. J., Dunlop, J. S., Baum, S. A., O'Dea, C. P., \& Hughes, D. H. 1999, MNRAS, 308, 377

McMahon, R. G., Priddey, R. S., Omont, A., Snellen, I., \& Withington, S. 1999, MNRAS, 309, L1

Menou, K., Haiman, Z., \& Narayanan, V. K. 2001, ApJ, 558, 535

Merritt, D. \& Ferrarese, L. 2001, MNRAS, 320, L30

Milosavljević, M. \& Merritt, D. 2001, ApJ, 563, 34

Milosavljević, M. \& Merritt, D. 2002, AAS/Division of Dynamical Astronomy Meeting, 33

Murphy, D. W., Browne, I. W. A., \& Perley, R. A. 1993, MNRAS, 264, 298

Neufeld, D. A. 1999, in After the Dark Ages: When Galaxies were Young (the Universe at $2<z<5$ ), ed. S. Holt and E. Smith (: AIPP), 414

Neugebauer, G., Green, R. F., Matthews, K., Schmidt, M., Soifer, B. T., \& Bennett, J. 1987, ApJS, 63, 615

Netzer, H. 2003, ApJ, 583, L5

Ohta, K., Yamada, T., Nakanishi, K., Kohno, K., Akiyama, M., \& Kawabe, R. 1996, Nature, 382, 426

Omont, A. 2003, preprint (astro-ph/0304340)

Omont, A., Cox, P., Bertoldi, F., McMahon, R. G., Carilli, C., \& Isaak, K. G. 2001, A\&A, 374, 371

Omont, A., Petitjean, P., Guilloteau, S., McMahon, R. G., Solomon, P. M., \& Pecontal, E. 1996, Nature, 382, 428

Onken, C. A. \& Peterson, B. M. 2002, ApJ, 572, 746

Osmer, P. S. 1982, ApJ, 253, 28

Osmer, P. S. 2003, Carnegie Observatories Astrophysics Series, Vol. 1: Coevolution of Black Holes and Galaxies, ed. L. C. Ho (Cambridge: Cambridge Univ. Press), in press (astro-ph/0304150)

Papadopoulos, P. P., Röttgering, H. J. A., van der Werf, P. P., Guilloteau, S., Omont, A., van Breugel, W. J. M., \& Tilanus, R. P. J. 2000, ApJ, 528, 626

Papovich, C., Dickinson, M., \& Ferguson, H. C. 2001, ApJ, 559, 620

Pei, Y. C. 1995, ApJ, 438, 623

Pentericci, L., Röttgering, H. J. A., Miley, G. K., McCarthy, P., Spinrad, H., van Breugel, W. J. M., \& Macchetto, F. 1999, A\&A, 341, 329

Pentericci, L., McCarthy, P. J., Röttgering, H. J. A., Miley, G. K., van Breugel, W. J. M., \& Fosbury, R. 2001, ApJS, 135, 63

Pentericci, L. et al. 2002, AJ, 123, 2151

Peterson, B. M. 1997, An introduction to active galactic nuclei (Cambridge: Cambridge University Press)

Peterson, B. M. 2002, in Active Galactic Nuclei: From Central Engine to Host Galaxy, ed. S. Collin, F. Combes, and I. Shlosman (San Francisco: ASP), 6

Peterson, B. M., Polidan, R. S., \& Pogge, R. W. 2001, ASP Conf. Ser. 224, Probing the Physics of Active Galactic Nuclei by Multiwavelength Monitoring (San Francisco: ASP)

Peterson, B. M. \& Wandel, A. 1999, ApJ, 521, L95

Peterson, B. M. \& Wandel, A. 2000, ApJ, 540, L13
Peterson, B. M., et al. 2000, ApJ, 542, 161

Petric, A. O., Carilli, C. L., Bertoldi, F., Fan, X., Cox, P., Strauss, M. A., Omont, A., \& Schneider, D. P. 2003, AJ, 126, 15

Priddey, R. S. \& McMahon, R. G. 2001, MNRAS, 324, L17

Priddey, R. S., Isaak, K. G., McMahon, R. G., \& Omont, A. 2003, MNRAS, 339, 1183

Reuland, M., van Breugel, W., Röttgering, H., de Vries, W., De Breuck, C., \& Stern, D. 2003, ApJ, 582, L71

Richards, G. T. et al. 2001, AJ, 121, 2308

Ridgway, S. E., Heckman, T. M., Calzetti, D., \& Lehnert, M. 2001, ApJ, 550, 122

Rix, H.-W. Falco, E. E., Impey, C., Kochanek, C., Lehár, J., McLeod, B., Muñoz, J., \& Peng, C. 2001, ASP Conf. Ser. 237: Gravitational Lensing: Recent Progress and Future Goals, ed. T. G. Brainerd \& C. S. Kochanek (San Francisco: ASP), 169

Rowan-Robinson, M. 2000, MNRAS, 316, 885

Sanders, D. B., Phinney, E. S., Neugebauer, G., Soifer, B. T., \& Matthews, K. 1989, ApJ, 347, 29

Sanders, D. B., Scoville, N. Z., \& Soifer, B. T. 1991, ApJ, 370, 158

Schirber, M. \& Bullock, J. S. 2003, ApJ, 584, 110

Schlegel, D. J., Finkbeiner, D. P., Davis, M. 1998, ApJ, 500, 525

Schmidt, M., \& Green, R. F. 1983, ApJ, 269, 352

Schmidt, M., Schneider, D. P., \& Gunn, J. E. 1995, A.J, 110, 68

Schneider, D. P., Schmidt, M., \& Gunn, J. E. 1997, AJ, 114, 36

Schneider, D. P. et al. 2001, AJ, 121, 1232

Shaver, P. A., Wall, J. V., Kellermann, K. I., Jackson, C. A., \& Hawkins, M. R. S. 1996, Nature, 384, 439

Shields, J. C., Ferland, G. J., \& Peterson, B. M. 1995, ApJ, 441, 507

Silk, J., \& Rees, M. J. 1998, A\&A, 331, L1

Solomon, P. M., Downes, D., Radford, S. J. E., \& Barrett, J. W. 1997, ApJ, 478, 144

Steidel, C. C., Giavalisco, M., Pettini, M., Dickinson, M., \& Adelberger, K. L. 1996, ApJ, 462, L17

Steidel, C. C., Adelberger, K. L., Giavalisco, M., Dickinson, M., \& Pettini, M. 1999, ApJ, 519, 1

Steidel, C. C., Hunt, M. P., Shapley, A. E., Adelberger, K. L., Pettini, M., Dickinson, M., \& Giavalisco, M. 2002, ApJ, 576, 653

Stern, D., Djorgovski, S. G., Perley, R. A., de Carvalho, R. R., Wall, J. V. 2000, ApJ, 119, 1526

Storrie-Lombardi, L. J., McMahon, R. G., Irwin, M. J., \& Hazard, C. 1996, ApJ, 468, 121

Tacconi, L. J., Genzel, R., Tecza, M., Gallimore, J. F., Downes, D., \& Scoville, N. Z. 1999, ApJ, 524, 732

Theuns, T., Mo, H. J., \& Schaye, J. 2001, MNRAS, 321, 450

Turner, E. L. 1991, AJ, 101, 5

van Bemmel, I. M. \& Dullemond, C. P. 2003, A\&A, 404, 1

van Breugel, W. J. M., Stanford, S. A., Spinrad, H., Stern, D., \& Graham, J. R. 1998, ApJ, 502, 614

Vestergaard, M. 2000, PASP, 112, 1504

Vestergaard, M. 2002, ApJ, 571, 733 (paper I)

Vestergaard, M., \& Wilkes, B. J. 2001, ApJS, 134, 1

Vestergaard, M., Wilkes, B. J., \& Barthel, P. D. 2000, ApJ, 538, L103

Vignali, C., Brandt, W. N., Schneider, D. P., Garmire, G. P., \& Kaspi, S. 2003, AJ, 125, 418

Volonteri, M., Haardt, F., Madau, P. 2002, ApJ, 582, 559

Wandel, A., Peterson, B. M., \& Malkan, M. A. 1999, ApJ, 526, 579

Warren, S. J., Hewett, P. C., \& Osmer, P. S. 1994, ApJ, 421, 412

Wilkes, B. J., Kuraszkiewicz, J., Green, P. J., Mathur, S., McDowell, J. C. 1999a, ApJ, 513, 76

Wilkes, B. J., Hooper, E. J., McLeod, K. K., Elvis, M. S., Impey, C. D., Lonsdale, C. J., Malkan, M. A., \& McDowell, J. C. 1999b, ESA SP-427: The Universe as Seen by ISO, 845

Willott, C. J., McLure, R. J., \& Jarvis, M. J. 2003a, ApJ, 587, L15

Willott, C. J., Rawlings, S., Jarvis, M. J., \& Blundell, K. M. 2003b, MNRAS, 339, 173

Wyithe, J. S. B. \& Loeb, A. 2002, ApJ, 577, 57

Yi, I. 1996, ApJ, 473, 645

Yuan, W., Brinkmann, W., Siebert, J., \& Voges, W. 1998, A\&A, 330, 108

Yun, M. S., Carilli, C. L., Kawabe, R., Tutui, Y., Kohno, K., \& Ohta, K. 2000, ApJ, 528, 171 